Peop 1 of 2

Proj.

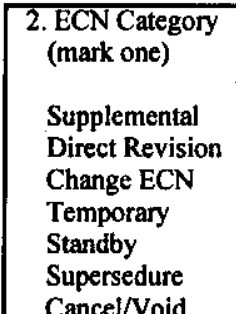

3. Originator's Name, Organization, MSIN, and Telephone No.

S. H. Peck, Nuclear Safety, R3-26, 372-3641

6. Project Title/No./Work Order No.

SNF Project K Basins

9. Document Numbers Changed by this ECN (includes sheet no. and rev.)

HNF-3960 Rev. 1

\begin{tabular}{|l|l|}
$\begin{array}{l}\text { 4. USQ Required? } \\
{[\mathrm{x}] \text { Yes [] No }}\end{array}$ & $\begin{array}{l}\text { 5. Date } \\
\mathbf{8} / 22 / \phi \phi\end{array}$ \\
\hline $\begin{array}{c}\text { 7. Bldg./Sys./Fac. No. } \\
\text { K Basins }\end{array}$ & $\begin{array}{c}\text { 8. Approval Designator } \\
\mathrm{S}^{\mathrm{N}}\end{array}$ \\
\hline N/A & N/A $/$ Related ECNNo(s). \\
\hline
\end{tabular}

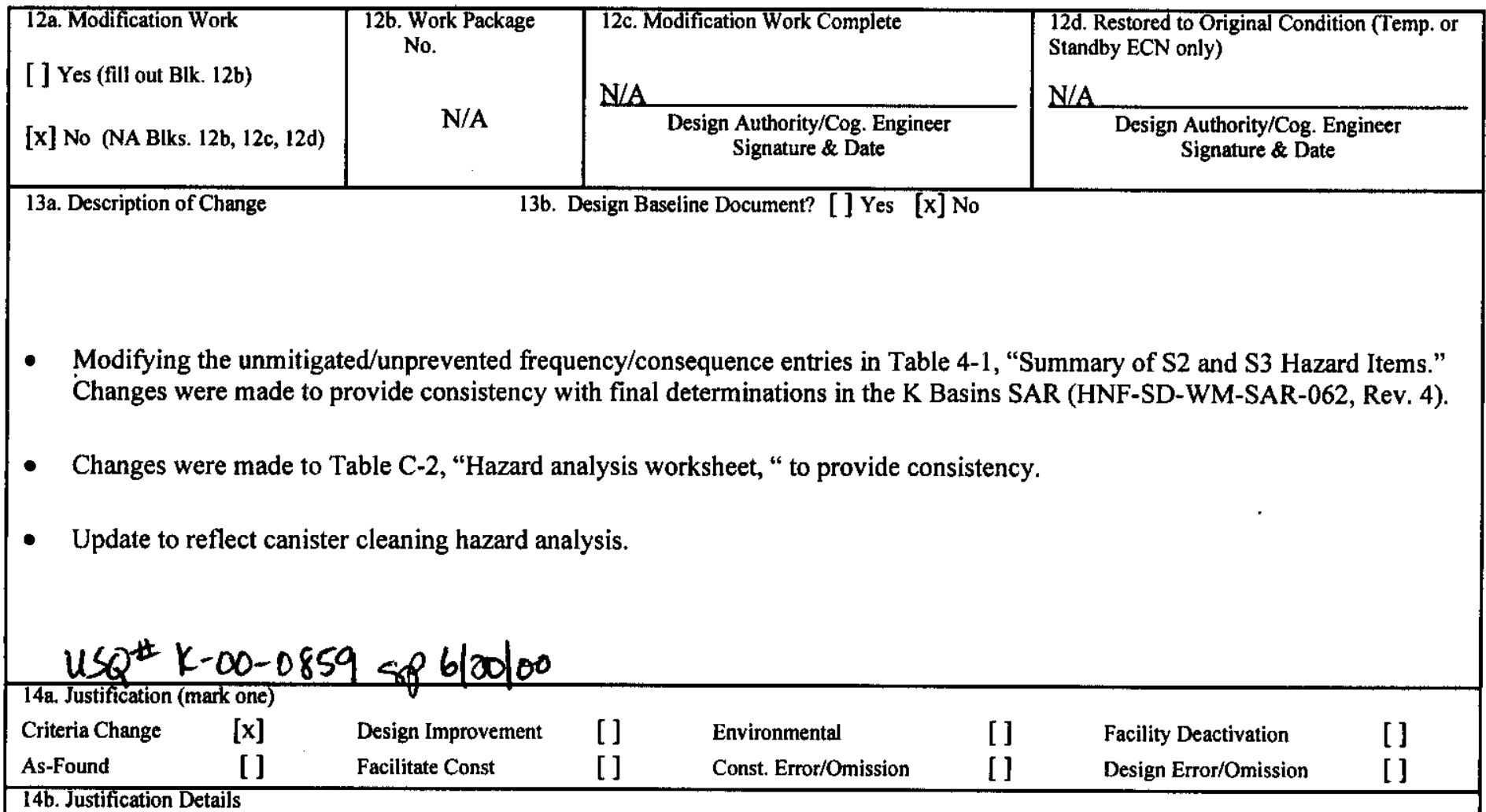

14b. Justification Details

The K Basins Fire Hazards Analysis (FHA) (HNF-SD-SNF-FHA-001, Rev. 1) has been issued and the changes made in this document are provided for consistency with the FHA.

15. Distribution (include name, MSIN, and no. of copies)
M. J. Langevin
$\mathrm{X} 3-76$
C. T. Miller
X3-79
S. H. Peck
R3-26
J. H. Wicks
X3-71
B. J. Craig
R3-26
K Basins Project Files
$\mathrm{X} 3-90$
SNF Project Files
R3-11

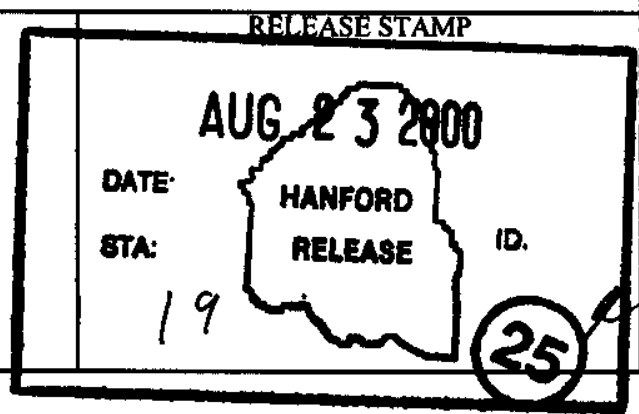




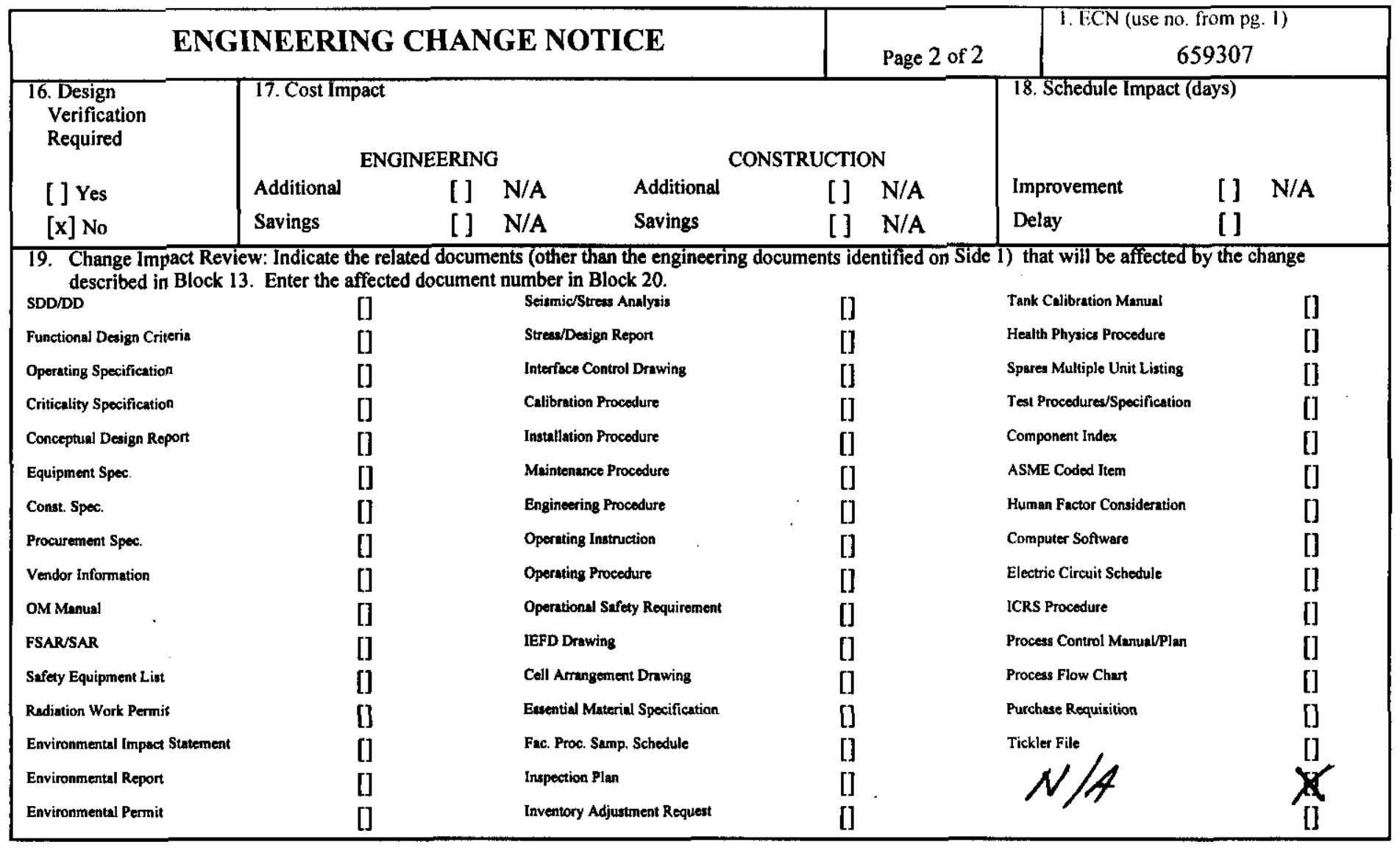

20. Other Affected Documents: (NOTE: Documents listed below will not be revised by this ECN.) Signatures below indicate that the signing organization has been notified of other affected documents listed below.

Note: These changes are being made to reflect the K Basin SAR, Rev. 4 or do not effect the SAR.

21. Approvals

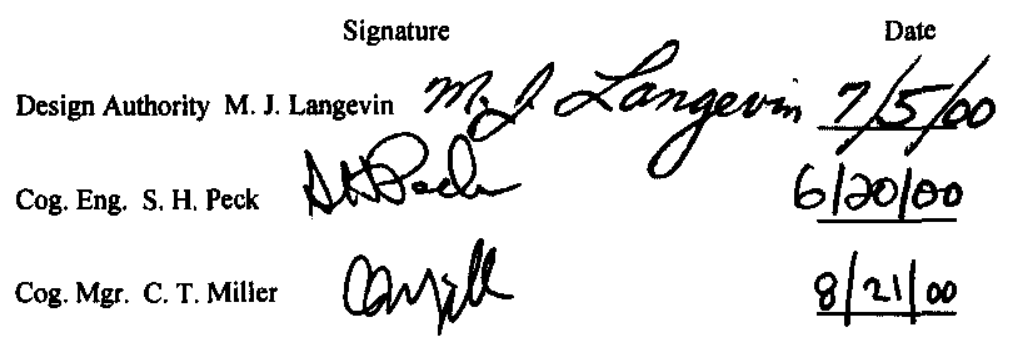

QA N/A

Safety C. T. Miller
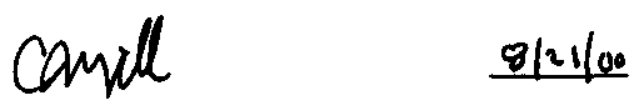

Environ. N/A

Operations J. H. Wicks

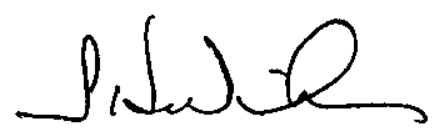

DEPARTMENT OE ENERGX

Signature or a Control Number that tracks the Approval Signature

$22 A 062000$ ADDIrIONAL 
HNF-3960

Revision 2

\section{K Basin Hazard Analysis}

Prepared for the U.S. Department of Energy Assistant Secretary for Environmental Management

Project Hanford Management Contractor for the

U.S. Department of Energy under Contract DE-AC06-96RL13200

Fluor Hanford

P.0. Box 1000

Richland, Washington 
HNF-3960

Revision 2

ECN 659307

\section{K Basin Hazard Analysis}

Document Type: HBD

Division: SNF

S.H. Peck

Mid Columbia Engineering

Date Published

August 2000

Prepared for the U.S. Department of Energy

Assistant Secretary for Environmental Management

Project Hanford Management Contractor for the

U.S. Department of Energy under Contract DE-AC06-96RL13200

Fluor Hanford

P.O. Box 1000

Richland, Washington
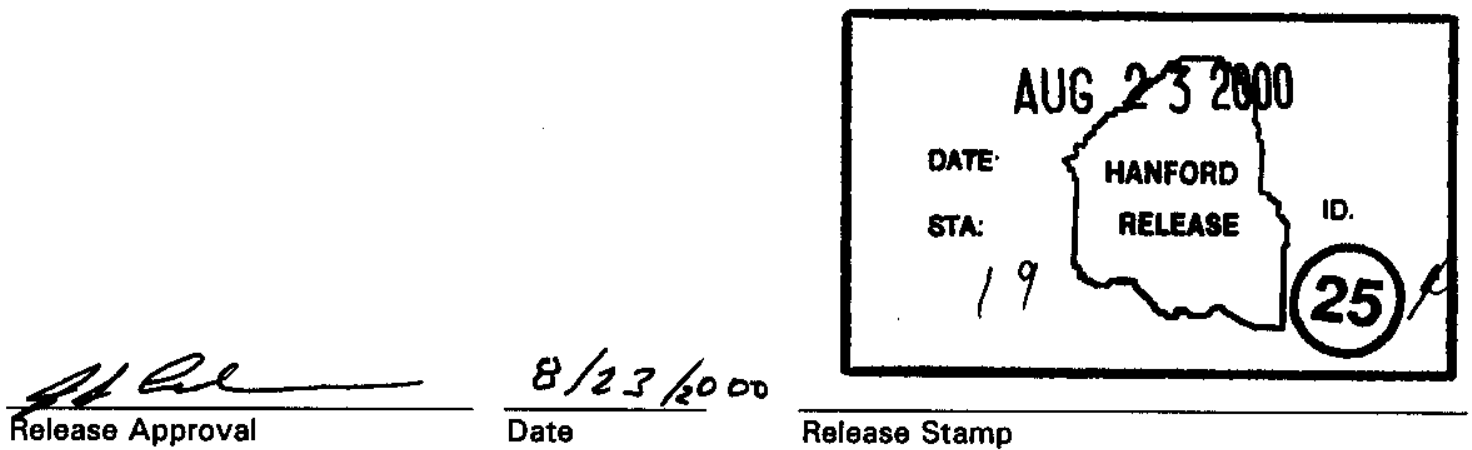

Release Stamp 


\section{RECORD OF REVISION}

(1) Document Number

HNF-3960, Rev. 2

Page 1

(2) Title

K Basin Hazard Analysis

\begin{tabular}{|c|c|c|c|}
\hline \multicolumn{4}{|c|}{ CHANGE CONTROL RECORD } \\
\hline \multirow[t]{2}{*}{ (3) Revision } & \multirow[t]{2}{*}{ (4) Description of Change - Replace, Add, and Delete Pages } & \multicolumn{2}{|c|}{ Authorized for Release } \\
\hline & & (5) Cog. Engr. Date & (6) Cog. Mgr. \\
\hline 0 & EDT: 626877 Original Document & $\begin{array}{l}\text { R. H. Webb } \\
9 / 20 / 99\end{array}$ & $\begin{array}{l}\text { R. L. Garrett } \\
9 / 20 / 99\end{array}$ \\
\hline 1 & 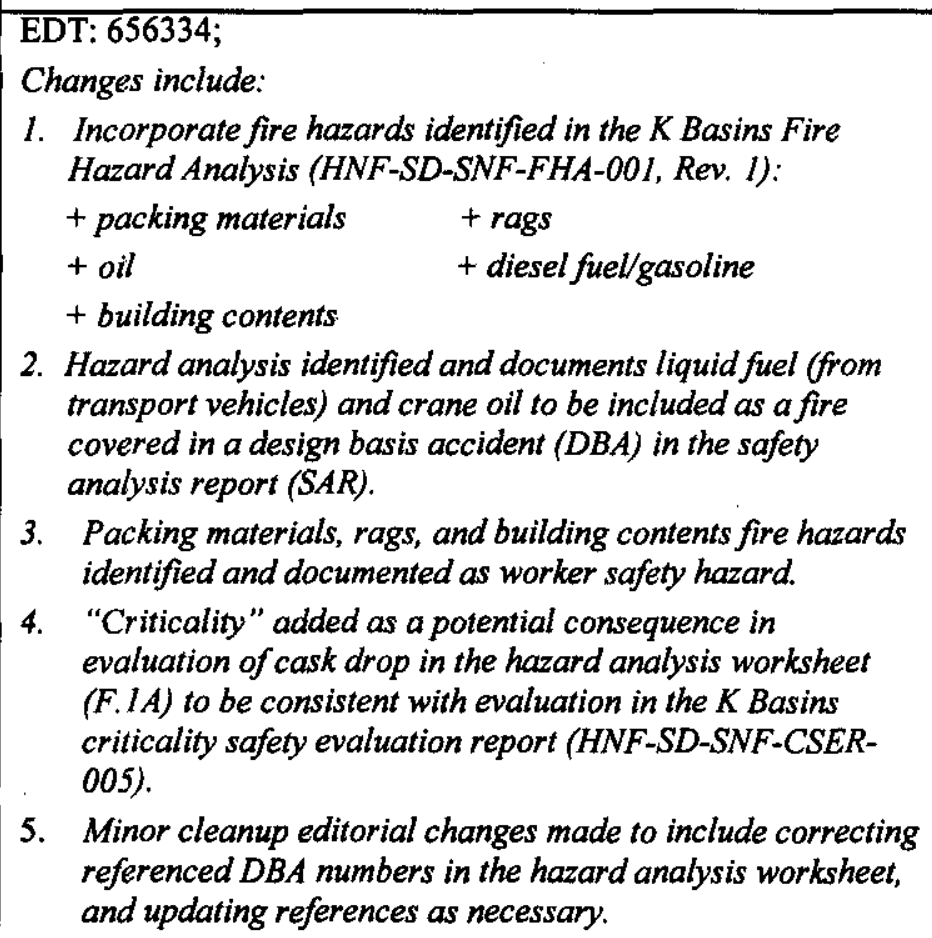 & $\begin{array}{l}\text { R. H. Webb } \\
12 / 17 / 99\end{array}$ & $\begin{array}{l}\text { R. L. Garrett } \\
12 / 28 / 99\end{array}$ \\
\hline 2 & $\begin{array}{l}\text { ECN: } 659307 \\
\text { Changes include: } \\
\text { I. Modifying the unmitigated /unprevented frequency/ } \\
\text { consequence entries in Table 4-1, "Summary of S2 and S3 } \\
\text { Hazard Items." Changes were made to provide consistency } \\
\text { with final determinations in the K Basins SAR (HNF-SD- } \\
\text { WM-SAR-062, Rev. 4). } \\
\text { 2. Changes were made to Table C-2, "Hazard Analysis } \\
\text { Worksheet," to provide consistency. } \\
\text { 3. Update to reflect canister cleaning hazard analysis. }\end{array}$ & $6 / 20 / 00$ & $\begin{array}{l}\text { c. T. Miller } \\
8 / 21 / \infty\end{array}$ \\
\hline & & & \\
\hline & & & \\
\hline & & & \\
\hline & & & \\
\hline
\end{tabular}


HNF-3960

Revision 2

TRADEMARK DISCLAIMER

Reference herein to any specific commercial product, process,

or service by trade name, trademark, manufacturer, or

otherwise, does not necessarily constitute or imply its

endorsement, recommendation, or favoring by the United

States Government or any agency thereof or its contractors or subcontractors.

This report has been reproduced from the best available copy.

Printed in the United States of America

Total Pages: $\quad 63$ 
Abstract: This report describes the methodology used in conducting the $\mathrm{K}$ Basins Hazard Analysis, which provides the foundation for the K Basins Final Safety Analysis Report. This hazard analysis was performed in accordance with guidance provided by DOE-STD-3009-94, Preparation Guide for U. S. Department of Energy Nonreactor Nuclear Facility Safety Analysis Reports and implements the requirements of DOE Order 5480.23, Nuclear Safety Analysis Report.

Keywords: Hazard, Frequency, Consequence, Source, Radiation, Prevention, Mitigation, Worker, Safety, Abnormal Event 
HNF-3960

Revision 2

\section{K BASIN HAZARD ANALYSIS}

HNF-3960

Revison 2

June 2000 
HNF-3960 REV 2

This page intentionally left blank. 


\section{CONTENTS}

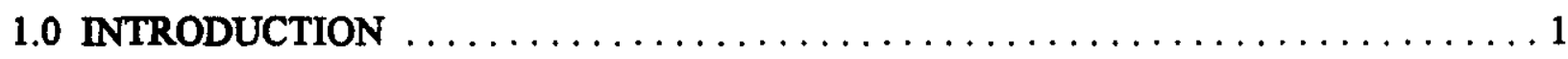

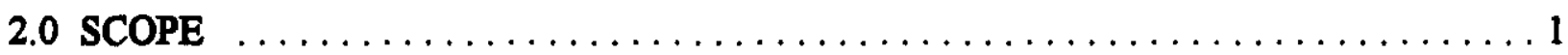

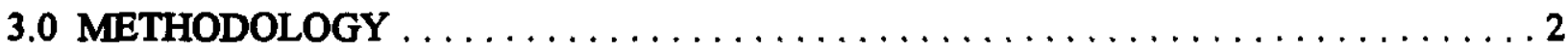

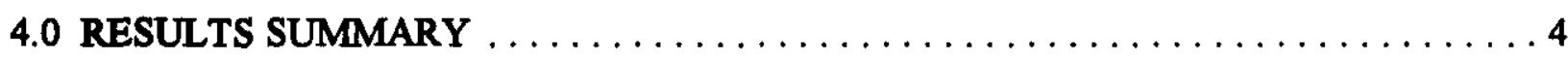

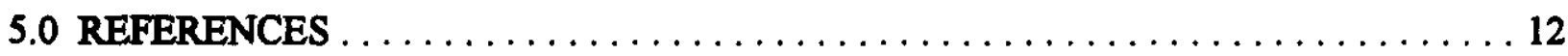

Attachment A: Hazardous Material List $\ldots \ldots \ldots \ldots \ldots \ldots \ldots \ldots \ldots \ldots \ldots \ldots$

Attachment B: Hazard Baseline - Energy Checklist $\ldots \ldots \ldots \ldots \ldots \ldots \ldots \ldots \ldots$

Attachment C: Hazard Analysis Worksheet $\ldots \ldots \ldots \ldots \ldots \ldots \ldots \ldots \ldots \ldots \ldots$

Attachment D: K Basin Hazard Analysis Team Members . . . . . . . . . . . . . D-1

\section{LIST OF TABLES}

3-1. Qualitative Accident Severity Levels $\ldots \ldots \ldots \ldots \ldots \ldots \ldots \ldots \ldots \ldots$

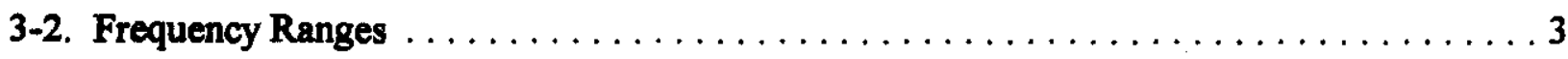

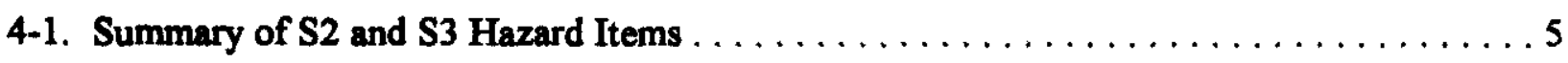

4-2. Summary of $\mathbf{S 1}$ (Worker Safety) Hazards $\ldots \ldots \ldots \ldots \ldots \ldots \ldots \ldots \ldots$

4-3. Hazard Initiators for Potential Abnormal Event for $\mathrm{K}$ Basins $\ldots \ldots \ldots \ldots \ldots$ 
HNF-3960 REV 2

\section{LIST OF TERMS}

\begin{tabular}{|ll|}
\hline ALARA & as low as reasonably achievable \\
DBA & design basis accident \\
DBE & design basis earthquake \\
DOE & U.S. Department of Energy \\
FRS & Fuel Retrieval System \\
HPT & health physics technician \\
IPSS & immersion pail support structure \\
IWTS & Integrated Water Treatment System \\
IXM & ion-exchange module \\
KE & K East \\
KW & K West \\
MCO & Multi-Canister Overpack \\
NLOP & north loadout pit \\
SAR & safety analysis report \\
SLOP & south loadout pit \\
SNF & spent nuclear fuel \\
ISR & technical safety requirement
\end{tabular}


Purolite ${ }^{T M}$ is a registered trademark of Brotech Corporation, Cywyd, Pennsylvania.

Magnafloc ${ }^{\mathrm{TM}}$ is a registered trademark of Ciba Speciality Chemicals Water Treatment Limited, West Yorkshire, England

Americoat ${ }^{\mathrm{TM}}$ is a registered trademark of Americoat Corporation, Brea, Ca.

Delo ${ }^{\mathrm{TM}}$ is a registered trademark of standard Oil Company, San Francisco, Ca

Regal Oil R\&OTM is a registered trademark of Texaco InC., New York, New York.

Jetlube ${ }^{\text {TM }}$ is a registered trademark of Jet-lube corp., Houston, Texas.

WD-40 ${ }^{\mathrm{TM}}$ is a registered trademark of WD-40 Company, San Diego, Ca.

Amberlite ${ }^{T M}$ is a registered trademark of Resinous Products \& Chemical Company, Philadelphia, Pennsylvania

Krylon ${ }^{T M}$ is a registered trademark of Krylon, Inc., Norristown, Pennsylvania.

Lectraclean ${ }^{\mathrm{TM}}$ is a registered trademark of CRC Industries, Inc., Warminster, Pennsylvania.

Dectol ${ }^{T M}$ is a registered trademark of Continental oil Corporation, Ponca City, Oklahoma 


\subsection{INTRODUCTION}

The K East (KE)/K West (KW) Basins in the $100 \mathrm{~K}$ Area of the Hanford Site have been used for storage of irradiated N Reactor and single-pass reactor fuel. The spent fuel is currently being stored underwater in racks and canisters in the basins. The Spent Nuclear Fuel (SNF) Project is adding equipment to the facility in preparation for removing the fuel and sludge from the basins.

In preparing this hazard analysis, a variety of hazard analysis techniques were used by the K Basins hazard analysis team, including hazard and operability studies, preliminary hazard analyses, and "what if' analyses (WHC-SD-SNF-PHA-001, HNF-2032, HNF-2456, and HNF-SD-SNF-SAD-002). This document summarizes the hazard analyses performed as part of the safety evaluations for the various modification projects and combines them with the original hazard analyses to create a living hazard analysis document. As additional operational activities and modifications are developed, this document will be updated as needed to ensure it covers all the hazards at the K Basins in a summary form and to ensure the subsequent safety analysis is bounding. This hazard analysis also identifies the preliminary set of design features and controls that the facility could rely on to prevent or reduce the frequency or mitigate consequences of identified accident conditions based on their importance and significance to safety. The operational controls and institutional programs relied on for prevention or mitigation of an uncontrolled release are identified as potential technical safety requirements.

All operational activities and energy sources at the $\mathrm{K}$ Basins are evaluated in this hazard analysis. Using a systematic approach, this document identifies hazards created by abnormal operating conditions and external events (e.g., earthquakes) that have the potential for causing undesirable consequences to the facility worker, the onsite individual, or the public.

\subsection{SCOPE}

The systems and operational activities associated with the $\mathrm{KE}$ and $\mathrm{KW}$ Basins and support buildings were evaluated for potential hazards that could endanger facility workers or result in unacceptable releases of radioactive or hazardous chemical materials, which could affect the environment or the public. The scope of operations includes continued storage, retrieval, repackaging, and loadout of the SNF and debris. This hazard analysis will be updated to reflect future SNF analyses, such as sludge removal, to ensure all hazards are included.

The related support buildings evaluated include the following:

- $165 \mathrm{KE}$ - Power Control Building

- 1717 K Building - Maintenance Shop

- 183.1 KW Chlorine Vault (Spent Ion-Exchange Column Storage) 


\section{HNF-3960 REV 2}

- Spent Ion-Exchange Module (IXM) Storage Pads

- $1706 \mathrm{KE}$ and KEL - Water Studies Semiworks Facility, Development Laboratory

- $190 \mathrm{KE}$ - Main Pump House

- $183 \mathrm{KE}$ - Clearwells, Filters, Sedimentation Basins, Headhouse, Chlorine Vault

- $165 \mathrm{KW}$ - Switchgear

- $1724 \mathrm{~K}$ - Maintenance Shop Addition

- 185 K - Package Water Treatment Plant.

\subsection{METHODOLOGY}

The hazard identification process systematically and comprehensively identifies hazards that can endanger facility workers or cause unacceptable releases of radioactive or hazardous chemical materials, which can affect the environment or the public. The hazard analysis process (1) identifies hazardous conditions, (2) determines causes, and preventive and mitigative features, and (3) qualitatively estimates the consequences and frequencies of occurrence. Results of the hazard analysis are used to select candidate accidents for quantitative analysis in the $\mathrm{K}$ Basin Safety Analysis Report (SAR).

As part of the hazard identification process, a hazardous materials identification table (Attachment A, Table A-1) was developed from the Containment Vessel Collection Forms (HNF-3262). An energy source checklist, adapted from DOE-76-45-19, Job and Task Analysis, and HNF-PRO-704, Hazard and Accident Analysis Process, was also used to identify energy sources. The completed energy source checklist can be found in Attachment B, Table B-1.

The energy source checklist was evaluated to identify hazards not controlled by standard industrial safety programs or other institutional programs (e.g., Radiation Protection Program). These hazards were then entered into the hazard analysis table for additional analysis. Each hazardous condition was evaluated to identify the following:

- Potential accidents arising from the presence of the hazard

- Potential causes and consequences of the accident

- Design features or administrative controls credited to prevent the condition or mitigate the accident consequences

- Estimates of the likelihood and consequences of the accident.

Additional defense-in-depth features that can prevent or mitigate the accident consequences or frequency, or provide worker protection, are also included. The completed hazard analysis is included as Table C-1 in Attachment $\mathrm{C}$. 


\section{HNF-3960 REV 2}

The impacts of the potential unmitigated accidents developed in the hazard analysis worksheet were qualitatively ranked with respect to the frequency and severity of the consequences. Consequences were ranked in order of increasing severity, as shown in Table 3-1. Descriptions of the frequency rankings are included in Table 3-2.

Table 3-1. Qualitative Accident Severity Levels.

\begin{tabular}{|c|l|}
\hline Consequence Assessment Code & \multicolumn{1}{|c|}{ Description } \\
\hline S3 & $\begin{array}{l}\text { There is sufficient material and energy available to cause a } \\
\text { high or moderate impact to the maximum off-site individual. }\end{array}$ \\
\hline S2 & $\begin{array}{l}\text { There is sufficient material and energy available to cause a } \\
\text { high or moderate impact to the maximum on-site individual. }\end{array}$ \\
\hline S1 & $\begin{array}{l}\text { There is sufficient material and energy available to cause an } \\
\text { industrial injury, radiological dose, or chemical exposure to } \\
\text { one or more facility workers. }\end{array}$ \\
\hline S0 & $\begin{array}{l}\text { There is insufficient material and energy to adversely impact } \\
\text { facility workers. }\end{array}$ \\
\hline
\end{tabular}

Table 3-2. Frequency Ranges.

\begin{tabular}{|c|l|c|}
\hline Frequency Assessment & \multicolumn{1}{|c|}{ Description } & \multicolumn{1}{|c|}{ Estimated Frequency } \\
\hline F3 & $\begin{array}{l}\text { Has occurred or is likely to occur during } \\
\text { the lifetime of the facility. }\end{array}$ & $\begin{array}{c}\text { Anticipated: } \\
1.0 \mathrm{E}-02 / \mathrm{yr} \leq \mathrm{F} 3<1.0 \mathrm{E}-01 / \mathrm{yr}\end{array}$ \\
\hline F2 & $\begin{array}{l}\text { Is foresceable, but unlikely to occur } \\
\text { during the lifetime of the facility. }\end{array}$ & $\begin{array}{c}\text { Unlikely: } \\
1.0 \mathrm{E}-04 / \mathrm{yr} \leq \mathrm{F} 2<1.0 \mathrm{E}-02 / \mathrm{yr}\end{array}$ \\
\hline F1 & $\begin{array}{l}\text { Is perhaps possible, but extremely } \\
\text { unlikely to occur during the lifetime of } \\
\text { the facility. }\end{array}$ & $\begin{array}{c}\text { Extremely Unlikely: } \\
1.0 \mathrm{E}-06 / \mathrm{yr} \leq \mathrm{F} 1<1.0 \mathrm{E}-04 / \mathrm{yr}\end{array}$ \\
\hline F0 & $\begin{array}{l}\text { Is considered too improbable to warrant } \\
\text { further consideration. }\end{array}$ & $\begin{array}{c}\text { Beyond extremely unlikely: } \\
\text { F0 }<1.0 \mathrm{E}-06 / \mathrm{yr}\end{array}$ \\
\hline
\end{tabular}




\section{HNF-3960 REV 2}

\subsection{RESULTS SUMMARY}

As stated in DOE-STD-3009-94, Preparation Guide for U.S. Department of Energy Nonreactor Nuclear Facility Safety Analysis Reports, "the final purpose of the hazard analysis is to identify a limited subset of accidents to be carried forward to accident analysis." The initial selection of hazard items requiring further quantitative considerations was based on consequence only. Only items ranked as $\mathbf{S 3}$ or $\mathbf{S 2}$ were considered for evaluation as design basis accidents. Results of this screening are shown in Table 4-1. Events that were categorized as S2/F1 were not further considered, unless they were identified to have fatality impacts to the facility worker. Frequency categories for each event were estimated to assist in subsequent safety analysis.

Table 4-2 identifies hazards associated with the facility workers. These hazards do not necessarily warrant detailed accident analysis in the K Basins SAR as they are controlled by worker safety or other institutional control programs.

Four hazards analyses (WHC-SD-SNF-PHA-001, HNF-2032, HNF-2456, and HNF-SD-SNF-SAD-002) were combined and updated to create this hazard analysis. Other hazard analyses, such as the hazard analysis for canister cleaning, have been included by direct revision. The defined hazards were reviewed for hazards that could be initiators for "off-normal or abnormal operations" and warrant brief coverage in the K Basins SAR. Abnormal events are defined as operating conditions resulting from situations outside of normal operations, where normal operations are defined by process flow diagrams, system design descriptions, and operation and maintenance procedures. Consequences from abnormal events are typically standard industrial hazards that may include worker exposure to ionizing radiation. Events having radiological consequences greater than allowed by the facility radiological protection and ALARA (as low as reasonably achievable) programs do not fit the abnormal event profile and are required to be analyzed as accidents by the U.S. Department of Energy (DOE) safety analysis process. Abnormal events, their means of detection, consequences, and potential corrective actions are identified in Table 4-3. 
Table 4-1. Summary of S2 and S3 Hazard Items (2 Sheets).

\begin{tabular}{|c|c|c|c|}
\hline $\begin{array}{l}\text { Initial } \\
\text { Consequence } \\
\text { Eotimate }\end{array}$ & ID & Energy Source & Hazard Condition/Potential Accident \\
\hline $\mathbf{S} 2 / \mathrm{F}_{2}$ & B.1 & $\begin{array}{l}\text { Nuclear } \\
\text { Criticality }\end{array}$ & Load drops, seismic events, misloading, etc. \\
\hline $\mathrm{S} 3 / \mathrm{F} 2$ & C.1 & $\begin{array}{l}\text { Kineticl } \\
\text { Linear }\end{array}$ & $\begin{array}{l}\text { Truck and trailer movement of the cask-MCO, or moving forklift } \\
\text { or highlifter }\end{array}$ \\
\hline $\mathrm{S} 2 / \mathrm{F} 2$ & C. $2 \mathrm{~A}$ & $\begin{array}{l}\text { Kinetic/ } \\
\text { Linear }\end{array}$ & $\begin{array}{l}\text { Transfer bay crane moving cask/IXM; cask/XXM or hook catches } \\
\text { gantry structure }\end{array}$ \\
\hline $\mathbf{S} 2 / \mathbf{F}_{2}$ & C.2B & $\begin{array}{l}\text { Kinetic/ } \\
\text { Linear }\end{array}$ & $\begin{array}{l}\text { Transfer bay crane moving cask/LXM; cask/IXM strikes } \\
\text { NLOP/SLOP causing basin damage }\end{array}$ \\
\hline $\mathbf{S} 2 / \mathrm{F} 1$ & E.1 & $\begin{array}{l}\text { Pressure/ } \\
\text { Volume }\end{array}$ & $\begin{array}{l}\text { Mis-installed MCO shield plug (vent port closed); exceeds 24-hour } \\
\text { shipping window (note: no avenue exists for dose to go offsite) }\end{array}$ \\
\hline $\mathbf{S 2 / F 2}$ & E.2 & $\begin{array}{l}\text { Pressure/ } \\
\text { Volume }\end{array}$ & $\begin{array}{l}\text { Pressure in vessel, pumps, or piping; IXMs, Annular Filter Vessel, } \\
\text { above-water piping causes spray release }\end{array}$ \\
\hline S1/F2 & E.3 & $\begin{array}{l}\text { Pressure/ } \\
\text { Volume }\end{array}$ & $\begin{array}{l}\text { Pipe/valve failure of backup service water supply to basin causes } \\
\text { overflow of basin, spilling water (although S1, this event included } \\
\text { because it is a carry-over event that is included in the K Basins } \\
\text { SAR as a DBA) }\end{array}$ \\
\hline S3/F2 & E.4 & $\begin{array}{l}\text { Pressure/ } \\
\text { Volume }\end{array}$ & $\begin{array}{l}\text { Piping failure or mis-operation results in pumping water out of the } \\
\text { basin (note: water could get to river and offsite) }\end{array}$ \\
\hline $\mathbf{S} 2 / \mathrm{F} 3$ & F.1 & $\begin{array}{l}\text { Mass, Gravity, } \\
\text { Height }\end{array}$ & $\begin{array}{l}\text { Heavy load drop damages basin boundary (excluding drain valves) } \\
\text { (note: frequency before crediting lift devices and other SSCs that } \\
\text { would prevent damage to basin) }\end{array}$ \\
\hline $\mathrm{S} 2 / \mathrm{F} 2$ & F.1A & $\begin{array}{l}\text { Mass, Gravity, } \\
\text { Height }\end{array}$ & $\begin{array}{l}\text { Cask-MCO dropped: into IPSS, onto IPSS, onto IPSS bottom } \\
\text { plate, onto SLOP curb, onto operations deck and then tip-and-hit } \\
\text { the SLOP curb }\end{array}$ \\
\hline $\mathrm{S} 2 / \mathrm{F3}$ & F.2 & $\begin{array}{l}\text { Mass, Gravity, } \\
\text { Height }\end{array}$ & $\begin{array}{l}\text { Load drop/seismic forces damage the basin drain valves resulting in } \\
\text { loss of water (note: frequency before crediting drain valve covers) }\end{array}$ \\
\hline S3/F3 & F.3 & $\begin{array}{l}\text { Mass, Gravity, } \\
\text { Height }\end{array}$ & $\begin{array}{l}\text { Fuel containers or rack lifted out of water causing high radiation or } \\
\text { fuel fire (note: frequency before crediting safety-related lifting } \\
\text { devices) }\end{array}$ \\
\hline S3/F1 & F.4 & $\begin{array}{l}\text { Mass, Gravity, } \\
\text { Height, }\end{array}$ & $\begin{array}{l}\text { DBE causes failure of gantry while loading the MCO scrap basket } \\
\text { at the highest point; basin drains down causing high radiation and } \\
\text { fuel ignition }\end{array}$ \\
\hline $\mathbf{S 2 / F 1}$ & G.1 & $\begin{array}{l}\text { Flammable } \\
\text { Materials }\end{array}$ & $\begin{array}{l}\text { Hydrogen buildup results in hydrogen burn or explosion (Included } \\
\text { because of potential for worker fatality). }\end{array}$ \\
\hline S2/F2 & G.2 & $\begin{array}{l}\text { Flammable } \\
\text { Materials }\end{array}$ & $\begin{array}{l}\text { Liquid fuel (from either forklit, high-lifter, or MCO transport } \\
\text { tractor) spills and burns impacting transfer bay critical column. } \\
\text { Failure of a critical column could result in the transfer bay bridge } \\
\text { crane and supporting structural members dropping onto the annular } \\
\text { filter vessel. }\end{array}$ \\
\hline
\end{tabular}


HNF-3960 REV 2

Table 4-1. Summary of S2 and S3 Hazard Items (2 Sheets).

\begin{tabular}{|c|c|c|c|}
\hline $\begin{array}{c}\text { Inttial } \\
\text { Consequence } \\
\text { Entimate }\end{array}$ & ID & Energy Source & Hazard Condition/Potential Aceident \\
\hline $\mathrm{S} 3 / \mathrm{Fl}$ & G.3 & $\begin{array}{l}\text { Flammable } \\
\text { Materials }\end{array}$ & $\begin{array}{l}\text { Crane hydraulic oil leaks while performing operations above the } \\
\text { south loadout pit and oil pools around gantry column and burns. } \\
\text { Gantry fails and drops MCO basket, which perforates floor and } \\
\text { results in loss of basin water. }\end{array}$ \\
\hline S3/F2 & K.1 & Thermal & $\begin{array}{l}\text { Loss of cooling results in basin water heatup to beyond limit, } \\
\text { followed by MCO shipment and release }\end{array}$ \\
\hline $\mathrm{S} 3 / \mathrm{F} 1$ & L.1 & $\begin{array}{l}\text { Explosive } \\
\text { Pyrophoric }\end{array}$ & $\begin{array}{l}\text { Uranium hydrides accumulated in fuel canister causes rapid burn of } \\
\text { the fuel }\end{array}$ \\
\hline $\mathrm{S} 3 / \mathrm{Fl}$ & L.2 & $\begin{array}{l}\text { Explosive } \\
\text { Pyrophoric }\end{array}$ & $\begin{array}{l}\text { Buildup of metal uranium metal particles causes fuel fire in settler } \\
\text { or knockout pot; or air sparge of an annular filter vessel }\end{array}$ \\
\hline $\mathbf{S 3 / F 1}$ & L. 3 & $\begin{array}{l}\text { Explosive } \\
\text { Pyrophoric }\end{array}$ & Uranium metal/uranium hydrides burn due to dryout of basin \\
\hline S3/F2 & N.1 & $\begin{array}{l}\text { Natural } \\
\text { Phenomena }\end{array}$ & Earthquake (DBE) \\
\hline $\mathrm{S} 3 / \mathbf{F} 2$ & P.3 & Loss of Power & $\begin{array}{l}\text { General or partial power failure, reduced voltage; surge causes } \\
\text { cask-MCO to hang up on crane, exceeding shipping window and } \\
\text { causing release (note: detailed frequency calculation is found in } \\
\text { Appendix 3A of the K Basins SAR) }\end{array}$ \\
\hline
\end{tabular}

DBA $=$ design basis accident
DBE $=$ design basis earthquake
IPSS $=$ immersion pail support structure
IXM $=$ ion-exchange module
MCO $=$ multi-canister overpack
NLOP $=$ north loodout pit
SAR $=$ safety analysis report
SLOP $=$ south loadout pit


Table 4-2. Summary of S1 (Worker Safety) Hazards (2 sheets).

\begin{tabular}{|c|c|c|c|}
\hline $\begin{array}{l}\text { Initial } \\
\text { frequency } \\
\text { eatimate }\end{array}$ & $\mathbf{I D}^{\prime}$ & $\begin{array}{l}\text { Dnergy } \\
\text { source }\end{array}$ & Description \\
\hline F2 & W.E. 1 & Pressure-Volume & $\begin{array}{l}\text { Fuel Retrieval System flow in addition to recirculation pump } \\
\text { bypass to DXMs caused by misvalving air monitoring } \\
\text { equipment or incorrect cartridge filter start up after } \\
\text { replacement, causing a decrease in the basin water quality } \\
\text { resulting in increased worker exposure }\end{array}$ \\
\hline F2 & W.E. 2 & Pressure-Volume & $\begin{array}{l}\text { Pipe break, clogged filter, or improper valving causes low or } \\
\text { no flow resulting in contamination or increased worker } \\
\text { exposure }\end{array}$ \\
\hline F2 & W.E.3 & Pressure-Volume & $\begin{array}{l}\text { Misloading of MCO (too many scrap baskets, excess fuel } \\
\text { fines) causes cask to be pressurized during loadout, resulting } \\
\text { in potential of contamination release through cask seals }\end{array}$ \\
\hline F2 & W.E.4 & Pressure-Volume & $\begin{array}{l}\text { Inadequate helium purge of loaded MCO during preshipping } \\
\text { processing causes cask to be pressurized during loadout, } \\
\text { resulting in potential of contamination release through cask } \\
\text { seals }\end{array}$ \\
\hline F3 & W.F. $1^{2}$ & $\begin{array}{l}\text { Mass, gravity, } \\
\text { height }\end{array}$ & $\begin{array}{l}\text { Equipment drops in pool creating a splash resulting in an } \\
\text { aerosol release and ALARA problems during recovery }\end{array}$ \\
\hline F2 & W.F.2 & $\begin{array}{l}\text { Mass, gravity, } \\
\text { height }\end{array}$ & $\begin{array}{l}\text { Knockout pot too heavy when lifted resulting in equipment } \\
\text { damage, potential worker injury, and possible contamination of } \\
\text { the basin work area from splash }\end{array}$ \\
\hline F3 & W.F. $3^{2}$ & $\begin{array}{l}\text { Mass, gravity, } \\
\text { height }\end{array}$ & $\begin{array}{l}\text { Process dip tube not seated correctly prevents shield plug from } \\
\text { seating and results in a high radiation stream }\end{array}$ \\
\hline F3 & W.G.1 & $\begin{array}{l}\text { Flammable } \\
\text { material }\end{array}$ & $\begin{array}{l}\text { Combustible material (e.g., shipping crates, pallets, windbreak } \\
\text { material, impact-limiting foam, plastic containment tents, } \\
\text { anticontamination clothing) ignites and impacts critical } \\
\text { structural columns by direct flame impingement and hot gas } \\
\text { layer }\end{array}$ \\
\hline F1 & W.J.1 & Radiation & $\begin{array}{l}\text { Transfer of contaminated water to air lines, receiver, and } \\
\text { compressor results in additional exposure to worker }\end{array}$ \\
\hline F2 & W.J.2 & Radiation & $\begin{array}{l}\text { Waste pad and shipping dock store spent IXMs and packaged } \\
\text { cartridge filters (awaiting disposal or stored improperly) } \\
\text { resulting in increased worker exposure }\end{array}$ \\
\hline F2 & W.J.3 & Radiation & $\begin{array}{l}\text { Cs-137 trapped in piping and valves generates hot spots which } \\
\text { results in increased worker exposure }\end{array}$ \\
\hline F2 & W.J.4 & Radiation & $\begin{array}{l}\text { Loss of basin water level decreases shielding over the basin } \\
\text { source terms resulting in increased worker exposure }\end{array}$ \\
\hline F1 & W.M.1 & $\begin{array}{l}\text { Hazardous } \\
\text { material }\end{array}$ & $\begin{array}{l}\text { Loading wrong resin into IXM results in potential to increase } \\
\text { worker exposure }\end{array}$ \\
\hline
\end{tabular}


HNF-3960 REV 2

Table 4-2. Summary of S1 (Worker Safety) Hazards (2 sheets).

\begin{tabular}{|c|c|c|c|}
\hline $\begin{array}{c}\text { Initial } \\
\text { frequency } \\
\text { estimate }\end{array}$ & ID' & $\begin{array}{c}\text { Energy } \\
\text { source }\end{array}$ & \multicolumn{1}{c|}{ Description } \\
\hline F1 & W.P.1 & Loss of power & $\begin{array}{l}\text { Loss of radiation monitors result in the potential for workers } \\
\text { to receive increased exposure }\end{array}$ \\
\hline
\end{tabular}

${ }^{1}$ Event identifier is provided for cross-reference to Table C-2

${ }^{2}$ Hozards that are considered initiators of "abnormal events"

$\begin{array}{ll}\text { ALARA } & =\text { as low as reasonably achievable } \\ \text { IXM } & =\text { ion-exchange module } \\ \text { MCO } & =\text { multi-canister overpack } \\ \text { NLOP } & =\text { north loadout pit }\end{array}$




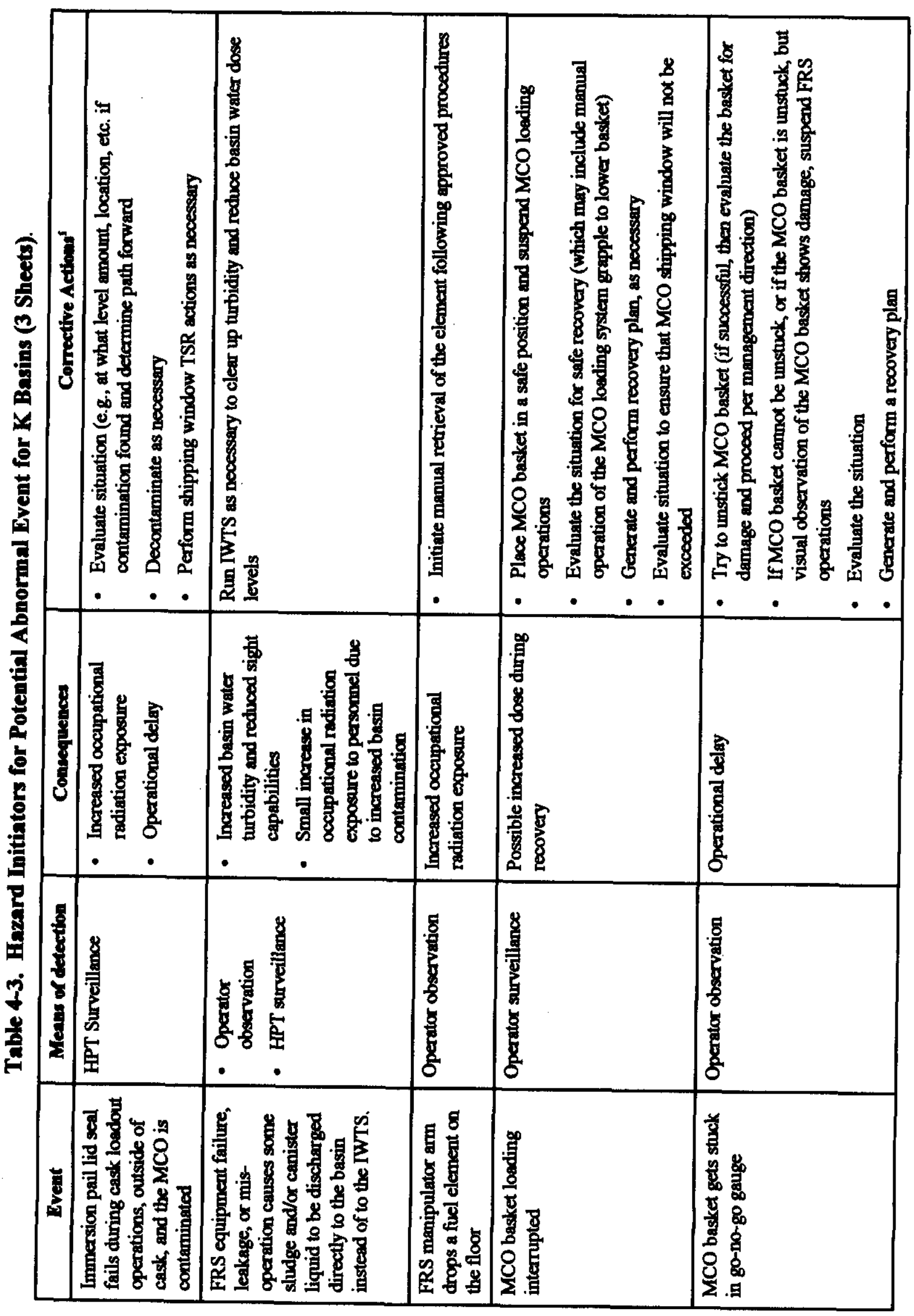


HNF-3960 REV 2

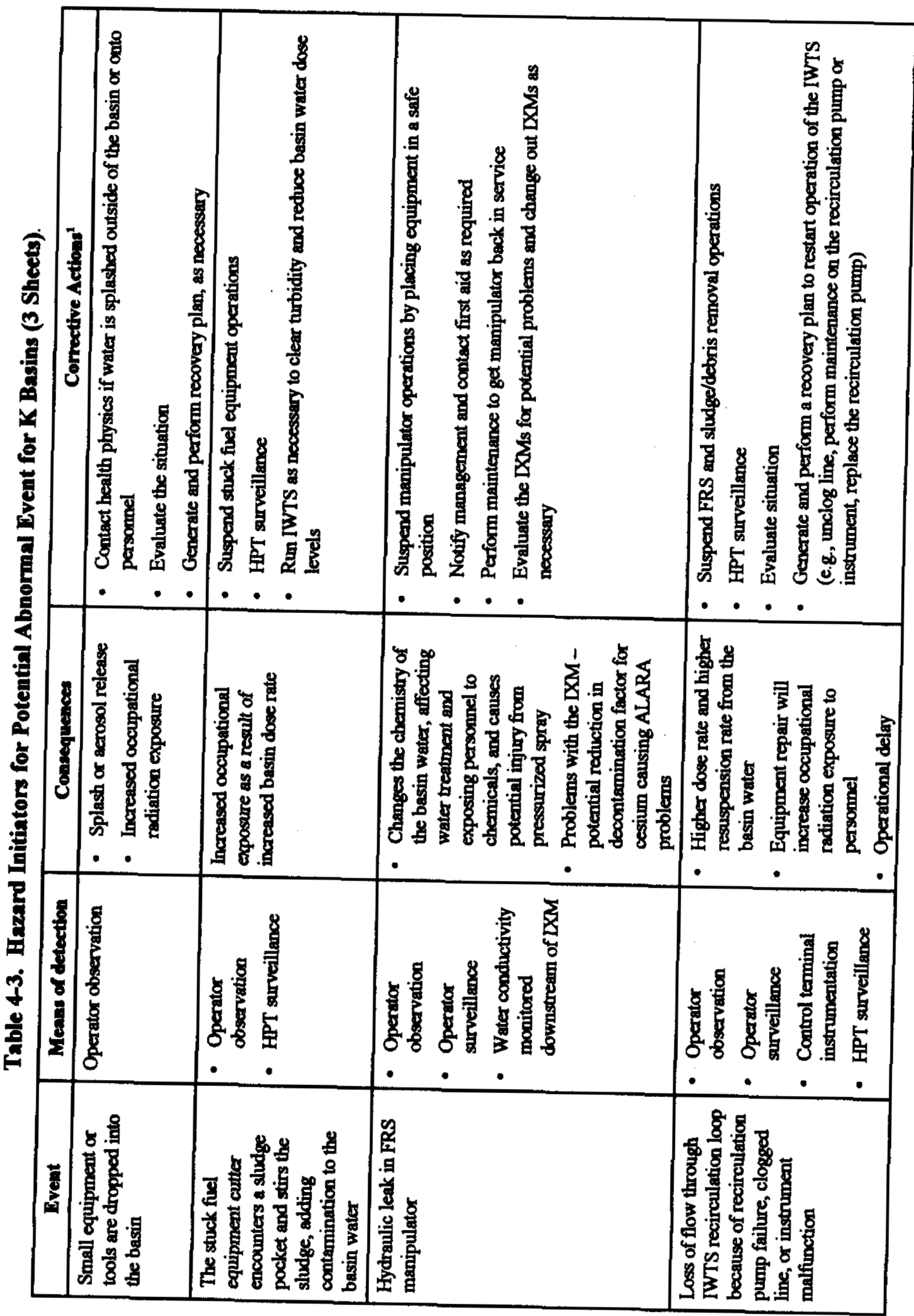




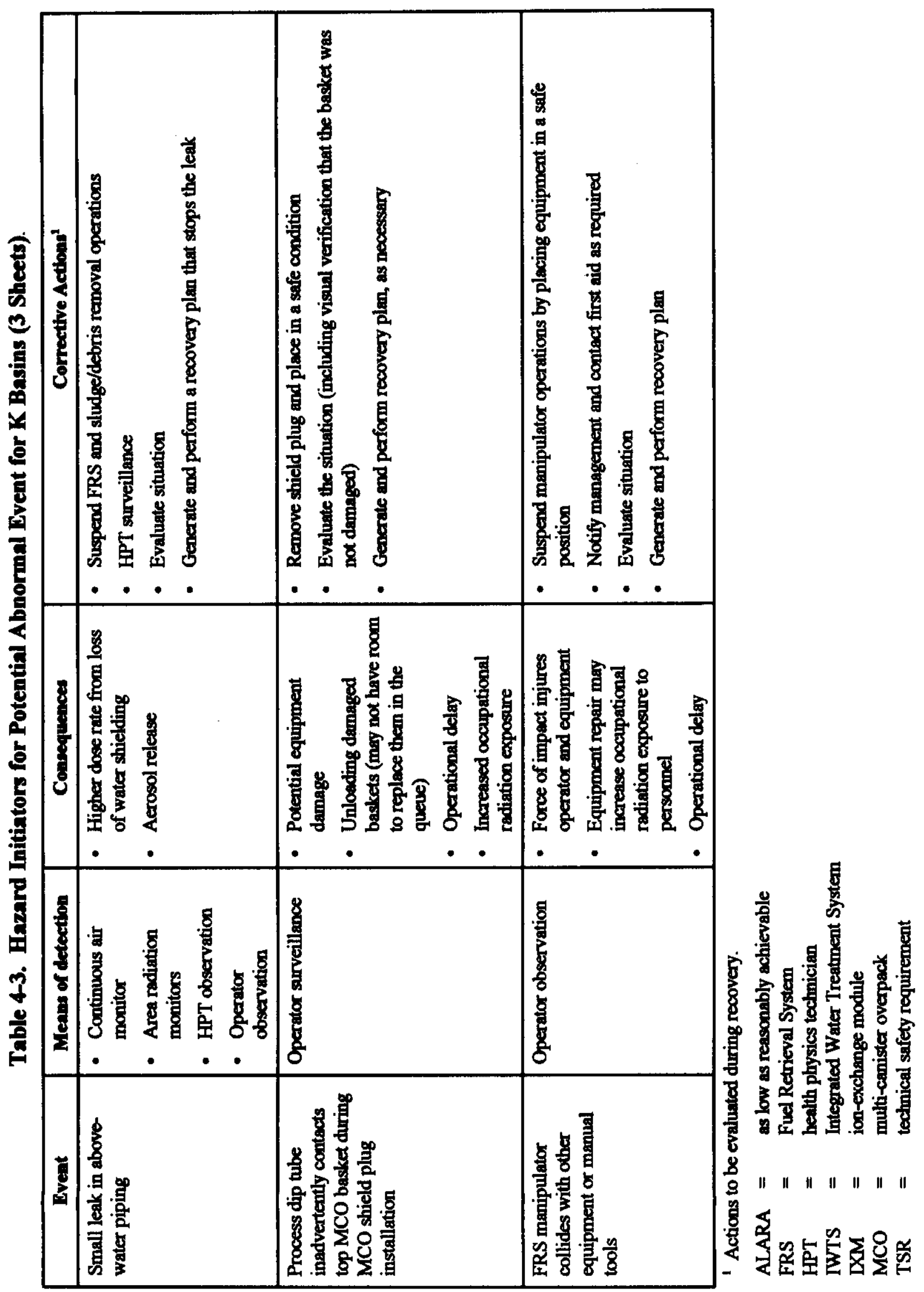


HNF-3960 REV 2

\subsection{REFERENCES}

DOE-76-45-19, 1979, Job and Task Analysis, U.S. Department of Energy, Washington, D.C.

DOE-STD-3009-94, 1994, Preparation Guide for U.S. Department of Energy Nonreactor Nuclear Facility Safety Analysis Reports, U.S. Department of Energy, Washington, D.C.

HNF-2032, 1998, SNF Fuel Retrieval Sub-Project Safety Analysis Document, Rev. 0, DE\&S Hanford, Richland, Washington.

HNF-2456, 1998, SNF Cask Loadout System Sub-Project Safety Analysis Document, DE\&S Hanford, Richland, Washington.

HNF-3262, 1998, Facility Vulnerability Assessment Phase 3 Final Report, Rev. 0, January 20, DE\&S Hanford, Inc., Richland, Washington.

HNF-PRO-704, Hazard and Accident Analysis Process, Fluor Hanford, Incorporated, Richland, Washington.

HNF-SD-SNF-SAD-002, 1998, Integrated Water Treatment System Subproject Safety Assessment Document, Rev. 2, DE\&S Hanford, Richland, Washington.

WAC 173-303, "Dangerous Waste Regulations," Washington Administrative Code, as amended.

WHC-SD-SNF-PHA-001, 1994, Preliminary Hazards Analysis of K-Basin Fuel Encapsulation and Storage, Rev. 0, Westinghouse Hanford Company, Richland, Washington.

\section{HAZARD ANALYSIS BASIS DOCUMENTS}

COGEMA-C-0105-RPT-003, 2000, Definitive Design Report: Spent Nuclear Fuels $K$ West Canister Cleaning Systems Sub-Project A.2.A, Rev. 0, COGEMA Engineering Corporation, Richland, Washington.

HNF-2032, 1998, SNF Fuel Retrieval Sub-Project Safety Analysis Document, Rev. 0, DE\&S Hanford, Richland, Washington.

HNF-2456, 1998, SNF Cask Loadout System Sub-Project Safety Analysis Document, DE\&S Hanford, Richland, Washington.

HNF-SD-SNF-SAD-002, 1998, Integrated Water Treatment System Subproject Safety Assessment Document, Rev. 2, DE\&S Hanford, Richland, Washington. 


\section{HNF-3960 REV 2}

HNF-SD-WM-SAR-062, 2000, K Basins Final Safety Analysis Report, Rev. 4, Fluor Hanford, Incorporated, Richland, Washington.

WHC-SD-SNF-PHA-001, 1994, Preliminary Hazards Analysis of K-Basin Fuel Encapsulation and Storage, Rev. 0, Westinghouse Hanford Company, Richland, Washington. 
HNF-3960 REV 2

This page intentionally left blank.

hnf-3960.r2 
HNF-3960 REV 2

Attachment A

HAZARDOUS MATERIAL LIST 
HNF-3960 REV 2

This page intentionally left blank 
Table A-1. Hazardous Material List (2 Sheets).

\begin{tabular}{|c|c|c|c|}
\hline & Hazardous Material & Location & Quantity \\
\hline $\begin{array}{l}105 \mathrm{KE} / \mathrm{KW} \\
\text { Basin systems }\end{array}$ & 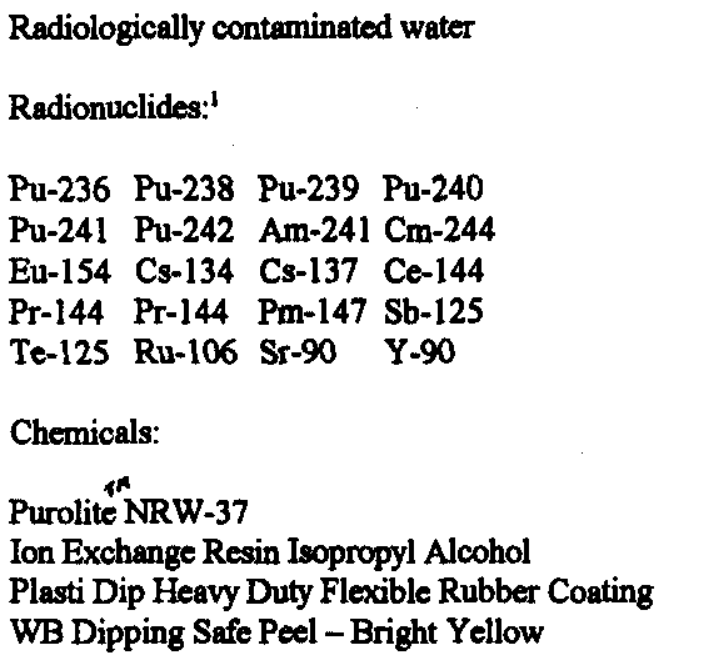 & $\begin{array}{l}105 \mathrm{KW} \\
105 \mathrm{KW} \\
105 \mathrm{KE} \\
105 \mathrm{KW}\end{array}$ & $\begin{array}{r}315 \mathrm{gal} \\
1 \mathrm{gal} \\
19 \mathrm{gal} \\
\sim 65 \mathrm{gal}\end{array}$ \\
\hline $\begin{array}{l}\text { Other } \\
\text { supporting } \\
\text { structures, } \\
\text { systems, and } \\
\text { components }\end{array}$ & $\begin{array}{l}\text { Radionuclides: } 39 \text { Spent IXCs } \\
\text { Chemicals: } \\
\text { Hydraulic Fluid (for FRS) } \\
\text { Liquid Propane } \\
\text { Dow Frost Heat Transfer Fluid } \\
\text { Sodium Bicarbonate } \\
\text { Liquid Alum (aluminum sulfate) } \\
\text { Lubricating Oil } \\
\text { Turbine Oil } \\
\text { Buffer Reagent for PC Hardness Analyzer } \\
\text { Purolite NRW-37 Ion Exchange Resin } \\
\text { Magnafloc } 990-N \\
\text { Sulfuric Acid } \\
\text { Valvoline Oil SAE30 } \\
\text { Amercoat } 90 \text { HS Resin } \\
\text { Deld } 400 \text { SAE } 40 \text { HD Motor Oil } \\
\text { Sodium Hydroxide } \\
\text { Turbine Oil } \\
\text { Indicator Solution for Pump Calorimeter Total Alka } \\
\text { Turbine Oil } \\
\text { Ethyl Alcohol, } 200 \text { proof } \\
\text { Coffing Transmiggion Oil } \\
\text { Dectol R\&O Oj' } 68 \text {, Conoco } \\
\text { Regal Oil R\&O } 220 \\
\text { Sili Kroj } \\
\text { Jet-Lube TFW }\end{array}$ & $\begin{array}{c}190 \mathrm{KE} \\
190 \mathrm{KE} \\
183 \mathrm{KE} \\
183 \mathrm{KE} \\
183 \mathrm{KE} \\
190 \mathrm{KE} \\
190 \mathrm{KE} \\
183 \mathrm{KE} \\
183.1 \mathrm{KW} \\
183 \mathrm{KE} \\
190 \mathrm{KE} \\
190 \mathrm{KE} \\
190 \mathrm{KE} \\
190 \mathrm{KE} \\
183 \mathrm{KE} \\
183 \mathrm{KE} \\
183 \mathrm{KE} \\
190 \mathrm{KE} \\
1717 \mathrm{~K} \\
1717 \mathrm{~K} \\
1717 \mathrm{~K} \\
1717 \mathrm{~K} \\
1717 \mathrm{~K} \\
1717 \mathrm{~K}\end{array}$ & $\begin{array}{c}\text { (2) } 55 \mathrm{gal} / \mathrm{drums} \\
500 \mathrm{gal} \\
807 \mathrm{gal} \\
90 \mathrm{gal} \\
\mathrm{empty} \\
12 \mathrm{gal} \\
25 \mathrm{gal} \\
3.5 \mathrm{gal} \\
39 \mathrm{IXCs} \\
1205 \mathrm{gal} \\
5 \mathrm{gal} \\
26 \mathrm{gal} \\
30 \mathrm{gal} \\
40 \mathrm{gal} \\
4 \mathrm{gal} \\
33 \mathrm{gal} \\
14 \mathrm{gal} \\
23 \mathrm{gal} \\
1 \mathrm{gal} \\
20 \mathrm{gal} \\
22 \mathrm{gal} \\
199 \mathrm{gal} \\
3 \mathrm{gal} \\
1 \mathrm{gal}\end{array}$ \\
\hline
\end{tabular}


Table A-1. Hazardous Material List (2 Sheets).

\begin{tabular}{|c|c|c|c|}
\hline & Haxardous Material & Location & Quantity \\
\hline $\begin{array}{l}\text { Other } \\
\text { supporting } \\
\text { structures, } \\
\text { systems, and } \\
\text { components } \\
\text { cont. }\end{array}$ & $\begin{array}{l}\text { Chemicals cont: } \\
\text { WD-40 } \\
\text { PPG Interior Enamel Semi-Gloss Acrylic Latex } \\
\text { Amberlite IR-120 Cation Exchange Resin } \\
\text { Krylon Spray Paint } \\
\text { Lectra Clean II } \\
\text { Sodium Sulfite } \\
\text { Lead } \\
\text { P-Bis (O-Methylstyryl)Benzene } \\
\text { Toluene } \\
\text { Toluene } \\
\text { Sulfuric Acid } \\
\text { Nitric Acid } \\
\text { Liquid Nitrogen } \\
\text { Isopropyl Alcohol } \\
\text { Sodium Hydroxide } \\
\text { Unleaded fuel, Conoco (in carbon steel tank) } \\
\text { WB Dipping Safe Peel - Bright Yellow }\end{array}$ & $\begin{array}{c}1717 \mathrm{~K} \\
1717 \mathrm{~K} \\
1706 \mathrm{KE} \\
165 \mathrm{KE} \\
165 \mathrm{KE} \\
1706 \mathrm{KEL} \\
\text { Recycle }^{4} \\
\mathrm{SAA}^{2} \\
\mathrm{SAA}^{2} \\
90 \mathrm{dSP}^{3} \\
\text { Recycle } \\
1706 \mathrm{KE} \\
\mathrm{Lab} \\
1705 \mathrm{KE} \\
1706 \mathrm{KE} \\
1717 \mathrm{KE} \\
190 \mathrm{KE}\end{array}$ & $\begin{array}{c}1 \mathrm{gal} \\
620 \mathrm{gal} \\
748 \mathrm{gal} \\
10 \mathrm{gal} \\
1.4 \mathrm{gal} \\
\text { less then } 1 \mathrm{gal}^{3} \\
20 \mathrm{ft}^{3} \mathrm{maximum} \\
1.5 \mathrm{gal} \\
20 \mathrm{gal} \\
55 \mathrm{gal} \\
20 \mathrm{ft}^{3} \text { maximum } \\
17 \mathrm{gal} \\
80 \mathrm{gal} \\
23 \mathrm{gal} \\
2 \mathrm{gal} \\
154 \mathrm{gal} \\
\sim 200 \mathrm{gal}\end{array}$ \\
\hline
\end{tabular}

1 HNF-SD-SNF-TI-015, 1998, Spent Nuclear Fuel Project Technical Databook, Rev. 6, DE\&S Hanford, Richland, Washington.

2 This is an established SAA and is under the control of the operator. The requirements for establishing this SAA are based on WAC-173-303, "Dangerous Waste Regulations," Washington Administrative Code, as amended, and PHMC procedure HNF-PRO-455, Solid Waste Management. Weekly inspections are performed and documented per HNF-PRO-455.

3 This is an established <90-day storage area and is under the control of the operator. The requirements for establishing, maintaining, and inspecting this <90-day storage area are based on WAC-173-303 and HNF-PRO-45S. The volume and percentage of primary chemicals fluctuate based on plant operations and quantities shipped to a TSD.

- This recycle staging area is for staging of lead acid batteries, non-PCB ballasts, aerosol cans, non-regulated oil, and fluorescent light bulbs. These materials are considered recycled and are shipped to the recycle consolidation center.

3 Quantity represents heel left in the bottom of a 50-gal tank.

FRS = Fuel Retrieval System
KE $=\mathbf{K}$ East
KW $=\mathbf{K}$ West
IXC $=$ ion-exchange column
PCB $=$ polychlorinated biphenyl
PHMC $=$ Project Hanford Management Contract
SAA $=$ satellite accumulation area
TSD = treatment, storage, and disposal facility




\section{HNF-3960 REV 2}

\section{Attachment B}

\section{HAZARD BASELINE - ENERGY CHECKLIST}




\section{HNF-3960 REV 2}

This page intentionally left blank. 
HNF-3960 REV 2

Table B-1. Hazard Baseline - Energy Checklist (13 Sheets).

\begin{tabular}{|c|c|c|c|c|}
\hline $\begin{array}{l}\text { Type of } \\
\text { Hevard }\end{array}$ & Form of Herard & 105 KE/KW Basin & $\begin{array}{c}\text { Other Supporting } \\
\text { Structurea, Syotems, and } \\
\text { Components }\end{array}$ & $\begin{array}{l}\text { Hounde } \\
\text { Requirine } \\
\text { Additond } \\
\text { Analyois }\end{array}$ \\
\hline \multirow{9}{*}{$\begin{array}{l}\text { A. Electrical } \\
\text { Note: All } \\
\text { clectrical hazands } \\
\text { are standard } \\
\text { industrial hazands } \\
\text { with the } \\
\text { exception of loss } \\
\text { of power, which } \\
\text { is covered by } \\
\text { external events } \\
\text { (see hazand P.4) }\end{array}$} & 1. Battery banks & $\mathbf{N} / \mathbf{A}$ & $\begin{array}{l}\text { Two battery rooms in } \\
\text { basement of } 165 \mathrm{KE} \text { (used } \\
\text { for switchgear, rectifiers and } \\
\text { distribution panols - part of } \\
\text { the DC nyctem) }\end{array}$ & None ${ }^{1}$ \\
\hline & 2. Diesel units & N/A & N/A & N/A \\
\hline & 3. High voltage lines & N/A & 230kV power poles & None ${ }^{1}$ \\
\hline & 4. Transformers & Power transformers & Power transformers & None $^{1}$ \\
\hline & 5. Wiring & AC/DC system & AC/DC system & None $^{1}$ \\
\hline & 6. Switchgear & $480 \mathrm{~V}$ switchgear at $105 \mathrm{KE} / \mathrm{KW}$ & $\begin{array}{l}230 \mathrm{kV} \text { switchgear at the } \\
151 \mathrm{~K} \text { substation } \\
13.8 \mathrm{kV} \text { switchgear in the } \\
165 \mathrm{~K} \text { Building } \\
480 \mathrm{~V} \text { switchgear } \\
\text { The system ends at the load } \\
\text { side of the motor control } \\
\text { center or at the secondary } \\
\text { side of the transformers for } \\
\text { voltages below } 480 \mathrm{~V}\end{array}$ & None' \\
\hline & $\begin{array}{l}\text { 7. Underground } \\
\text { wiring }\end{array}$ & N/A & Underground tunnel & None' \\
\hline & 8. Cable runs & Various cable raceways & Various cable raceways & None ${ }^{1}$ \\
\hline & $\begin{array}{l}\text { 9. Service outlets } \\
\text { and fittings }\end{array}$ & $\begin{array}{l}110 / 120 \mathrm{~V} \text { outlets are located } \\
\text { throughout the } 105 \mathrm{KE} / \mathrm{KW} \text { Basin } \\
\text { temporary welding receptacle } \\
\text { GFIs are located throughout the } \\
\text { basin }\end{array}$ & $\begin{array}{l}110 / 120 \mathrm{~V} \text { outlets } \\
\text { Temp welding receptacle }\end{array}$ & None' \\
\hline
\end{tabular}


HNF-3960 REV 2

Table B-1. Hazard Baseline - Energy Checklist (13 Sheets).

\begin{tabular}{|c|c|c|c|c|}
\hline $\begin{array}{l}\text { Type of } \\
\text { Heund }\end{array}$ & Form of Harand & 105 KT/KW Basin & $\begin{array}{c}\text { Other Supporting } \\
\text { Structures, Syotems, and } \\
\text { Components }\end{array}$ & $\begin{array}{l}\text { Henarde } \\
\text { Requiring } \\
\text { Addithonal } \\
\text { Andyol }\end{array}$ \\
\hline \multirow[t]{4}{*}{$\begin{array}{l}\text { A. Eloctrical } \\
\text { cont. }\end{array}$} & $\begin{array}{l}\text { 10. Electric motor } \\
\text { driven pumps }\end{array}$ & $\begin{array}{l}\text { Recirculation pump (3) } \\
\text { Booster pump (part of the IWTS } \\
\text { system) (KW only) } \\
\text { Skimmer pump } \\
\text { B-Sump pumps } \\
\text { C-Sump pumps } \\
\text { D-Sump pumps } \\
\text { Submerced pumps for the PCM, } \\
\text { decapper, and process table } \\
\text { High-pressure pump for the PCM } \\
\text { Hydraulic pump for the manipulator } \\
\text { Pump for the chiller } \\
\text { Sludge pumping equipment } \\
\text { (electrical submersible pump) } \\
\text { Boiler circulation pumps } \\
\text { Air conditioning pumps } \\
\text { Air compreseor } \\
\text { High-pressure pump for debris } \\
\text { cleaning }\end{array}$ & $\begin{array}{l}\text { River pumps: raw water } \\
\text { pumps (1500 HP motor) } \\
\text { Potable water pumps (3) } \\
\text { (300 HP motor) } \\
\text { Service water pumps } \\
\text { (300 HP) } \\
\text { Beckwath pumps (300 HP } \\
\text { motor) } \\
\text { Water treatment facility } \\
\text { pumps } \\
\text { Sample pumps } \\
\text { Outfall samplers } \\
\text { Outfall sample pumps/piping } \\
\text { Outfall temperature and flow } \\
\text { monitoring } \\
\text { Low lift pumps } \\
\text { Septic system pumps } \\
\text { Air compressor }\end{array}$ & None $^{1}$ \\
\hline & 11. Other motors & $\begin{array}{l}\text { PCM lid motor } \\
\text { Building exhaust fans } \\
\text { Decapper exhaust } \\
\text { Air exhauster/sampler motors } \\
\text { Motor 3-way valves } \\
\text { MLS gantry drive } \\
\text { Exhaust blowers (each basin has } \\
\text { four roof mountod) } \\
\text { Each basin has two evaporative } \\
\text { coolers used for summer conditions }\end{array}$ & $\begin{array}{l}\text { Control/valve room (165E) } \\
\text { contains most motor- } \\
\text { operatod valves for } \\
\text { raw/service water systems }\end{array}$ & None ${ }^{1}$ \\
\hline & 12. Heaters & $\begin{array}{l}\text { Hot water boilers } \\
\text { (8) Unit heaters } \\
\text { Heat pumps }\end{array}$ & $\begin{array}{l}\text { Unit heaters } \\
\text { Heat pumps } \\
\text { Water treatment structure } \\
\text { unit heaters }\end{array}$ & None ${ }^{1}$ \\
\hline & 13. Power tools & $\begin{array}{l}\text { Used during construction, } \\
\text { maintenanoc, and operations }\end{array}$ & $\begin{array}{l}\text { Used during construction, } \\
\text { maintenance, and operations }\end{array}$ & None ${ }^{1}$ \\
\hline
\end{tabular}


Table B-1. Hazard Baseline - Energy Checklist (13 Sheets).

\begin{tabular}{|c|c|c|c|c|}
\hline $\begin{array}{l}\text { Type of } \\
\text { Herend }\end{array}$ & Form of Hazard & $105 \mathrm{KE} / \mathrm{KW}$ Basin & $\begin{array}{l}\text { Other Supporting } \\
\text { Structures, Systems, and } \\
\text { Components }\end{array}$ & $\begin{array}{l}\text { Henards } \\
\text { Requirlne } \\
\text { Additional } \\
\text { Analyols }\end{array}$ \\
\hline \multirow[t]{2}{*}{$\begin{array}{l}\text { A. Eleotrical } \\
\text { cont. }\end{array}$} & 14. Hoists & $\begin{array}{l}\text { Various electric hoists are mounted } \\
\text { on manually and motor-operated } \\
\text { trolleys rated up to } 32 \text { ton } \\
\text { Tractor motors for hoist and flexible } \\
\text { tranafer crane }\end{array}$ & $\begin{array}{l}\text { 15-ton bridge crane } \\
\text { 25-ton bridge crane }\end{array}$ & None $^{1}$ \\
\hline & 16. Other & $\begin{array}{l}\text { Electrical roll-up doors } \\
\text { Instrumentation and cameras }\end{array}$ & $\begin{array}{l}\text { Traveling water intake screen } \\
\text { (motorized) } \\
\text { Raw water, bypass valve } \\
\text { cross tie (electric motorized) } \\
\text { Electrical roll-up doors }\end{array}$ & None ${ }^{1}$ \\
\hline \multirow{9}{*}{$\begin{array}{l}\text { B. Nucleas } \\
\text { Criticality }\end{array}$} & 1. Vaults & N/A & N/A & N/A \\
\hline & $\begin{array}{l}\text { 2. Temporary } \\
\text { storage areas }\end{array}$ & $\begin{array}{l}\text { Accumulated sludge storage: } \\
\text { KE weasel pit } \\
\text { KW dummy elevator pit } \\
\text { KE/KW sand filter beckwash pit } \\
\text { (NLOP) } \\
\text { KE/KW floor sludge } \\
\text { KW technical view pit } \\
\text { Water treatment: } \\
\text { Knockout pots (KW) } \\
\text { Particulate settler tanks (KW } \\
\text { weasel pit) } \\
\text { Annular filter vessel } \\
\text { IXMs, sand filters } \\
\text { Fuel storage: } \\
\text { Mnin basins } \\
\text { MCO basket queue }\end{array}$ & 39 spent $\mathrm{LXC} /(183.1 \mathrm{KW})$ & Yes \\
\hline & 3. Receiving areas & N/A & N/A & N/A \\
\hline & 4. Casks & $\begin{array}{l}\text { MCO, Chem-Nuclear Cask, PAS-1 } \\
\text { sumpling ceak }\end{array}$ & & Yes \\
\hline & 5. Burial grounds & N/A & N/A & N/A \\
\hline & 6. Storage tanks & N/A & $\mathbf{N} / \mathbf{A}$ & $\mathbf{N} / \mathbf{A}$ \\
\hline & 7. Storage racks & $\begin{array}{l}\text { Fuel storage racks on floor of each } \\
\text { besin }(\mathrm{KE} / \mathrm{KW}) \mathrm{MCO} \text { basket queue }\end{array}$ & N/A & Yes \\
\hline & 8. Canals and basins & $\mathbf{N} / \mathbf{A}$ & N/A & $\mathbf{N} / \mathbf{A}$ \\
\hline & 9. Decon solution & N/A & N/A & N/A \\
\hline
\end{tabular}


Table B-1. Hazard Baseline - Energy Checklist (13 Sheets).

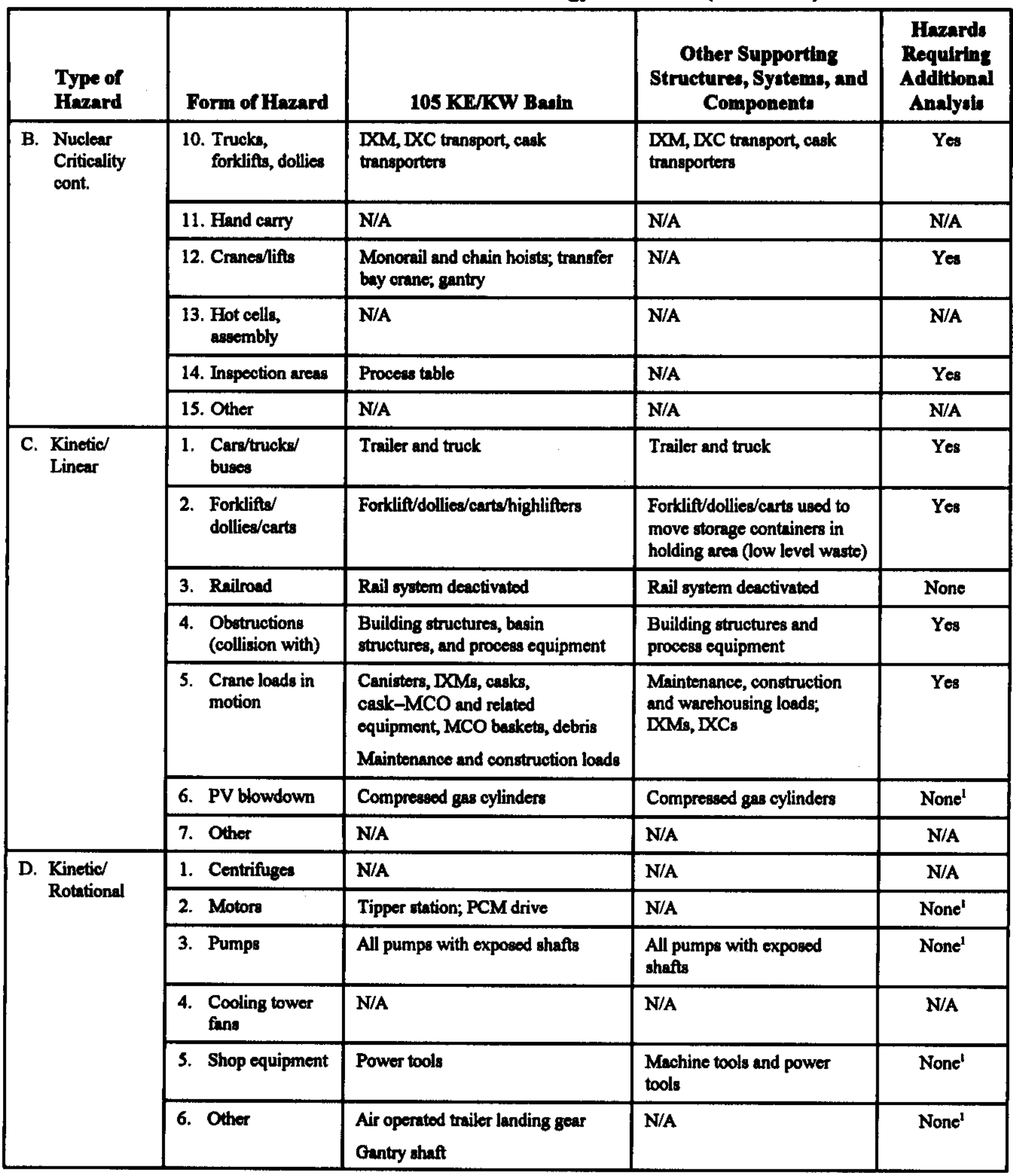


HNF-3960 REV 2

Table B-1. Hazard Baseline - Energy Checklist (13 Sheets).

\begin{tabular}{|c|c|c|c|c|}
\hline $\begin{array}{l}\text { Type of } \\
\text { Himand }\end{array}$ & Form of Herard & 105 KT/KW Basin & $\begin{array}{l}\text { Other Supporting } \\
\text { Structures, Systems, and } \\
\text { Components }\end{array}$ & $\begin{array}{l}\text { Merards } \\
\text { Requirias } \\
\text { Additional } \\
\text { Analyois }\end{array}$ \\
\hline \multirow{10}{*}{$\begin{array}{l}\text { E. Prowsure - } \\
\text { Volume }\end{array}$} & 1. Boilers & Package boilers - KE/KW & $\mathbf{N} / \mathbf{A}$ & None ${ }^{1}$ \\
\hline & $\begin{array}{l}\text { 2. Heated surge } \\
\text { tanks }\end{array}$ & Boiler expansion tank/air separator & N/A & None' \\
\hline & 3. Autoclaves & $\mathbf{N} / \mathbf{A}$ & N/A & $\mathbf{N} / \mathbf{A}$ \\
\hline & $\begin{array}{l}\text { 4. Test loops and } \\
\text { fncilities }\end{array}$ & $\mathbf{N} / \mathbf{A}$ & $\mathbf{N} / \mathbf{A}$ & $\mathbf{N} / \mathbf{A}$ \\
\hline & 5. Gas botties & $\begin{array}{l}\text { Inert gas system } \\
\text { Compreseed gas cylinders } \\
\text { SCBA } \\
\text { Various gas oylinders }\end{array}$ & Bottle gas system & None $^{1}$ \\
\hline & 6. Pressure vesseds & $\begin{array}{l}\text { Housing of cartridge filters vessel } \\
\text { Sand filter } \\
\text { DXMs } \\
\text { Air chilled evaporator } \\
\text { Knockout pots } \\
\text { Particulate settler vessel } \\
\text { Annular filter vessels } \\
\text { Compressed air vessel } \\
\text { MCO (once shield plug is installed) } \\
\text { Cask-MCO } \\
\text { Chem-Nuclear casks } \\
\text { PAS-l casks } \\
\text { Piping }\end{array}$ & $\mathbf{N} / \mathbf{A}$ & Yes \\
\hline & $\begin{array}{l}\text { 7. Streaced } \\
\text { members }\end{array}$ & $\mathbf{N} / \mathbf{A}$ & $\mathbf{N} / \mathbf{A}$ & $\mathbf{N} / \mathbf{A}$ \\
\hline & 8. Gas receivers & Shop air receiver & Shop air receivers & None $^{1}$ \\
\hline & $\begin{array}{l}\text { 9. Negative } \\
\text { pressure } \\
\text { collapise }\end{array}$ & N/A & $\mathbf{N} / \mathbf{A}$ & N/A \\
\hline & 10. Other & $\begin{array}{l}\text { Pressurized water wands; } \\
\text { Immersion pail tid seal } \\
\text { Trailer brakes }\end{array}$ & N/A & None $^{1}$ \\
\hline
\end{tabular}


HNF-3960 REV 2

Table B-1. Hazard Baseline - Energy Checklist (13 Sheets).

\begin{tabular}{|c|c|c|c|c|}
\hline $\begin{array}{l}\text { Type of } \\
\text { Hraned }\end{array}$ & Form of Harand & $105 \mathrm{KE} / \mathrm{KW}$ Banin & $\begin{array}{c}\text { Other Supportins } \\
\text { Structures, Systems, and } \\
\text { Components }\end{array}$ & $\begin{array}{l}\text { Henards } \\
\text { Requiring } \\
\text { Additional } \\
\text { Andyds }\end{array}$ \\
\hline \multirow[t]{13}{*}{$\begin{array}{l}\text { F. Mass, } \\
\text { Gravity, } \\
\text { Height }\end{array}$} & 1. Human effort & $\begin{array}{l}\text { Long-handled manual tools: } \\
\text { Manual tongs/air-operated tong } \\
\text { Hand-operated trolley system } \\
\text { Moving carts } \\
\text { Manully operated locking pin } \\
\text { on top of the IPSS } \\
\text { Maintenance, construction, and } \\
\text { operntions }\end{array}$ & $\begin{array}{l}\text { Maintenance, construction } \\
\text { and operations } \\
\text { Moving carts } \\
\text { Manual hoists }\end{array}$ & None ${ }^{1}$ \\
\hline & 2. Stairs & $\begin{array}{l}\text { Various building stairs } \\
\text { Operations platform } \\
\text { Cask loadout trailer stairs }\end{array}$ & $\begin{array}{l}\text { Various stairs } \\
\text { Water Treatment Facility }\end{array}$ & None' \\
\hline & 3. Lifts and cranes & $\begin{array}{l}\text { Canisters, knockout pots } \\
\text { DXMs, casks } \\
\text { Cask-MCO and related } \\
\text { equipment } \\
\text { MCO baskets, debris } \\
\text { Maintenance and construction loads }\end{array}$ & $\begin{array}{l}\text { Maintenance, construction } \\
\text { and warehousing loads } \\
\mathrm{LXMs}, \mathrm{XXCs} \\
\text { Low level waste containers }\end{array}$ & Yes \\
\hline & $\begin{array}{l}\text { 4. Bucket and } \\
\text { ladder }\end{array}$ & Maintenance activities & Maintenance activities & None' \\
\hline & 5. Trucks & $\begin{array}{l}\text { Truck deliveries in and out of the } \\
\text { transfer area }\end{array}$ & $\begin{array}{l}\text { Truck deliveries in other } \\
\text { areas }\end{array}$ & Yes \\
\hline & 6. Slings & $\begin{array}{l}\text { Construction, maintenance, and } \\
\text { operations }\end{array}$ & $\begin{array}{l}\text { Construction, maintenance, } \\
\text { and operations }\end{array}$ & None' \\
\hline & 7. Hoists & $\begin{array}{l}\text { Conatruction, maintenance, and } \\
\text { operation: }\end{array}$ & $\begin{array}{l}\text { Construction, maintenance, } \\
\text { and operations }\end{array}$ & Yes \\
\hline & 8. Elevators & N/A & N/A & $\mathbf{N} / \mathbf{A}$ \\
\hline & 9. Jecks & Truck jacks, pallet jacks & Truck jacks, pallet jacks & None' \\
\hline & $\begin{array}{l}\text { 10. Scafifolds and } \\
\text { ladders }\end{array}$ & $\begin{array}{l}\text { Construction, maintenance, and } \\
\text { operations }\end{array}$ & $\begin{array}{l}\text { Construction, maintenance, } \\
\text { and operations }\end{array}$ & None ${ }^{1}$ \\
\hline & $\begin{array}{l}\text { 11. Pits and } \\
\text { excavations }\end{array}$ & Basin and pits & Construction activities & None ${ }^{1}$ \\
\hline & 12. Elevated doors & N/A & N/A & N/A \\
\hline & 13. Vessels & $\mathbf{N} / \mathbf{A}$ & $\mathbf{N} / \mathbf{A}$ & N/A \\
\hline
\end{tabular}


Table B-1. Hazard Baseline - Energy Checklist (13 Sheets).

\begin{tabular}{|c|c|c|c|c|}
\hline $\begin{array}{l}\text { Type of } \\
\text { Trand }\end{array}$ & Form of Hward & $105 \mathrm{Ka} / \mathrm{KW}$ Basin & $\begin{array}{l}\text { Other Supporting } \\
\text { Structures, Syatems, and } \\
\text { Components }\end{array}$ & $\begin{array}{l}\text { Hemards } \\
\text { Requiring } \\
\text { Additional } \\
\text { Analyois }\end{array}$ \\
\hline $\begin{array}{l}\text { F. Mnas, } \\
\text { Gravity, } \\
\text { Height } \\
\text { cont. }\end{array}$ & 14. Other & $\begin{array}{l}\text { Building structure and grating } \\
\text { Rolt-up door } \\
\text { Deteriorating roof } \\
\text { FRS Manipulator drops }\end{array}$ & $\begin{array}{l}\text { Roll-up door } \\
\text { Deteriorating roof }\end{array}$ & Yes \\
\hline \multirow[t]{13}{*}{$\begin{array}{l}\text { G. Flammable } \\
\text { Mnterials }\end{array}$} & $\begin{array}{l}\text { 1. Packing } \\
\text { materials }\end{array}$ & $4 \times 4 \times 8$ shipping crates, pallets & $\begin{array}{l}4 \times 4 \times 8 \text { shipping crates, } \\
\text { pallets }\end{array}$ & Yes \\
\hline & 2. Rags & Cleaning rags and anti-c & Cleaning rags & Yes \\
\hline & 3. Gasoline & Forklfit, truck, and cranes & $\begin{array}{l}\text { Gasoline fucl storage: } \\
\text { Vehicles in parking lot }\end{array}$ & Yes \\
\hline & 4. Oil & $\begin{array}{l}\text { Chiller, crane, hoist } \\
\text { Manipulator hydraulic fluid }\end{array}$ & $\begin{array}{l}\text { Motor oil } \\
\text { Bearing lubrication } \\
\text { Oil storage }\end{array}$ & Yes \\
\hline & 5. Coolant oil & $\mathbf{N} / \mathbf{A}$ & N/A & N/A \\
\hline & 6. Paint solvent & Solvents and cleaners & Paints & None $^{2}$ \\
\hline & 7. Diesel fuel & $\begin{array}{l}\text { Fortlift used in waste sccumulation } \\
\text { area - } 30 \text { gal diesel fuel tank } \\
\text { Trucks in transfer area ( } 100 \mathrm{gal} \text { ) } \\
\text { Mobile crane for construction } \\
\text { purposes }\end{array}$ & Truck, forklift, mobile cranes & Yes \\
\hline & $\begin{array}{l}\text { 8. Buildings and } \\
\text { contents }\end{array}$ & $\begin{array}{l}\text { Building roofs, windbreak, } \\
\text { clectrical installation material } \\
\text { Canister cleaning enclosure }\end{array}$ & $\begin{array}{l}\text { Building roofs, wood } \\
\text { structures, clectrical } \\
\text { installation material }\end{array}$ & Yes \\
\hline & $\begin{array}{l}\text { 9. Trailers and } \\
\text { contents }\end{array}$ & $\begin{array}{l}\text { Personnel trailers (mobile offices), } \\
\text { vehicle tires }\end{array}$ & $\begin{array}{l}\text { Personnel trailers (mobile } \\
\text { office), vehicle tires }\end{array}$ & None ${ }^{2}$ \\
\hline & 10. Grease & $\begin{array}{l}\text { Small amount in } \\
\text { bearinga/gearboxes }\end{array}$ & $\begin{array}{l}\text { Small amount in } \\
\text { bearinge/gearboxes }\end{array}$ & None ${ }^{2}$ \\
\hline & $\begin{array}{l}\text { 11. Hydrogen } \\
\text { (including } \\
\text { bettery banks) }\end{array}$ & $\begin{array}{l}\text { Knockout pots } \\
\text { Canintern, basin sludge } \\
\text { Particulate settler } \\
\text { LXMs/DXC; cask-MCO } \\
\text { Annular filter vessels }\end{array}$ & $\begin{array}{l}\text { XXCs (183 KW)/battery } \\
\text { rooms each provided with } \\
\text { dedicated fans to ensure H } \\
\text { gas is released. }\end{array}$ & Yes \\
\hline & 12. Nitric acid & $\mathbf{N} / \mathbf{A}$ & $\mathbf{N} / \mathbf{A}$ & None' \\
\hline & 13. Organice & Decon solution & Decon solution & None ${ }^{2}$ \\
\hline
\end{tabular}


HNF-3960 REV 2

Table B-1. Hazard Baseline - Energy Checklist (13 Sheets).

\begin{tabular}{|c|c|c|c|c|}
\hline $\begin{array}{l}\text { Type of } \\
\text { Hrind }\end{array}$ & Form of Heand & $105 \mathrm{KD} / \mathrm{KW}$ Busin & $\begin{array}{c}\text { Other Supporting } \\
\text { Structures, Systems, and } \\
\text { Components }\end{array}$ & $\begin{array}{l}\text { Hozards } \\
\text { Requiring } \\
\text { Additional } \\
\text { Analysis }\end{array}$ \\
\hline \multirow[t]{3}{*}{$\begin{array}{l}\text { G. Flummable } \\
\text { Muteriala } \\
\text { cont. }\end{array}$} & 14. Gases - other & $\begin{array}{l}\text { Freon/chiller } \\
\text { Glycol/failure of basin heaters or } \\
\text { piping } \\
\text { P-10 gas used for portal monitors } \\
\text { Oxygen/acetylene }\end{array}$ & Oxygen/acetylene & None \\
\hline & 15. Spray paint & Maintenance use & Mnintenance use and storage & None $^{2}$ \\
\hline & 16. Other & Adjoining facility material storage & N/A & None ${ }^{2}$ \\
\hline \multirow[t]{6}{*}{ H. Corronive } & 1. Acida & Vehicle batteries & $\begin{array}{l}\text { Vehicle butteries } \\
\text { DC battery aystem }\end{array}$ & None $^{1}$ \\
\hline & 2. Caustics & N/A & Caustic cleaners & None' \\
\hline & $\begin{array}{l}\text { 3. "Natural" } \\
\text { chemicals (soil, } \\
\text { air, water) }\end{array}$ & N/A & N/A & N/A \\
\hline & 4. Decon solutions & Maintenance use & N/A & None ${ }^{1}$ \\
\hline & $\begin{array}{l}\text { 5. High } \\
\text { temperature } \\
\text { wuste }\end{array}$ & N/A & N/A & $\mathbf{N} / \mathbf{A}$ \\
\hline & 6. Other & Nitric acid & N/A & None' \\
\hline \multirow[t]{2}{*}{ J. Rediation } & 1. Canals & $\mathbf{N} / \mathbf{A}$ & N/A & N/A \\
\hline & 2. Plug storage & N/A & N/A & $\mathbf{N} / \mathbf{A}$ \\
\hline
\end{tabular}


HNF-3960 REV 2

Table B-1. Hazard Baseline - Energy Checklist (13 Sheets).

\begin{tabular}{|c|c|c|c|c|c|}
\hline & $\begin{array}{l}\text { Type of } \\
\text { Hevard }\end{array}$ & Form of Horand & 10S KR/KW Basin & $\begin{array}{c}\text { Other Supporting } \\
\text { Structurea, Systems, and } \\
\text { Components }\end{array}$ & $\begin{array}{l}\text { Henards } \\
\text { Requiring } \\
\text { Addftonal } \\
\text { Analyols }\end{array}$ \\
\hline & \multirow[t]{11}{*}{$\begin{array}{l}\text { Rediation } \\
\text { cont. }\end{array}$} & 3. Storage areas & $\begin{array}{l}\text { Accumulated sludge storage: } \\
\text { KE weasel pit } \\
\text { KW dummy elevator pit } \\
\text { KE/KW sand filter backwash } \\
\text { pit (NLOP) } \\
\text { KE/KW floor sludge } \\
\text { KW tochnical view pit } \\
\text { LXCs } \\
\text { Water treatment: } \\
\text { Knockout pots (KW) } \\
\text { Particulate settler tanks (KW } \\
\text { weasel pit) } \\
\text { Annular filter vessel } \\
\text { LXMs, sand filters, pumps and } \\
\text { piping } \\
\text { Fuel storage: } \\
\text { Canisters; MCO basket queue; } \\
\text { cask-MCO }\end{array}$ & Storage pad (DXMs) & Yes \\
\hline & & $\begin{array}{l}\text { 4. Storage } \\
\text { buildings }\end{array}$ & N/A & $183.1 \mathrm{KW}$ (DXC storage) & Yes \\
\hline & & $\begin{array}{l}\text { S. Radioactive } \\
\text { sources }\end{array}$ & Check sources & N/A & Nonel \\
\hline & & 6. Waste and scrap & $\begin{array}{l}\text { Waste accumulation areas in } \\
\text { transfer bay of each basin }\end{array}$ & Waste pad shipping dock & None \\
\hline & & 7. Contamination & $\begin{array}{l}\text { Contaminated water and } \\
\text { contaminated areas in both } \\
\text { K Basins }\end{array}$ & N/A & None ${ }^{1}$ \\
\hline & & $\begin{array}{l}\text { 8. Irradinted } \\
\text { experimental } \\
\text { and reactor } \\
\text { equipment }\end{array}$ & $\mathbf{N} / \mathbf{A}$ & $\mathbf{N} / \mathbf{A}$ & N/A \\
\hline & & 9. Electric furnaco & $\mathbf{N} / \mathbf{A}$ & N/A & N/A \\
\hline & & $\begin{array}{l}\text { 10. Blacklight (e.g., } \\
\text { magniflux) }\end{array}$ & $\mathbf{N} / \mathbf{A}$ & N/A & N/A \\
\hline & & 11. Laser & N/A & N/A & N/A \\
\hline & & 12. Modical X-ray & N/A & N/A & N/A \\
\hline & & $\begin{array}{l}\text { 13. Radiography } \\
\text { equipment and } \\
\text { sources }\end{array}$ & N/A & N/A & N/A \\
\hline
\end{tabular}


Table B-1. Hazard Baseline - Energy Checklist (13 Sheets).

\begin{tabular}{|c|c|c|c|c|}
\hline $\begin{array}{l}\text { Type of } \\
\text { Hezard }\end{array}$ & Form of Herard & $105 \mathrm{KW} / \mathrm{KW}$ Bain & $\begin{array}{c}\text { Other Supporting } \\
\text { Structures, Syotems, and } \\
\text { Components }\end{array}$ & $\begin{array}{l}\text { Hounde } \\
\text { Requiring } \\
\text { Additional } \\
\text { Analyal }\end{array}$ \\
\hline \multirow[t]{5}{*}{$\begin{array}{l}\text { J. Radiation } \\
\text { cont. }\end{array}$} & 14. Welding & Construction and maintenance & $\begin{array}{l}\text { Construction and } \\
\text { maintenance }\end{array}$ & None' \\
\hline & $\begin{array}{l}\text { 15. Electric arc, } \\
\text { other (high } \\
\text { current circuits) }\end{array}$ & $\mathbf{N} / \mathbf{A}$ & N/A & N/A \\
\hline & 16. Electron beam & $\mathbf{N} / \mathbf{A}$ & N/A & N/A \\
\hline & $\begin{array}{l}\text { 17. Equipment } \\
\text { noise }\end{array}$ & Pumps, motors, power tools, etc. & $\begin{array}{l}\text { Pumps, motors, power tools, } \\
\text { etc. } \\
\text { Noise from venting of raw } \\
\text { water pumps (tunnel) }\end{array}$ & None $^{1}$ \\
\hline & $\begin{array}{l}\text { 18. Ultrasonic } \\
\text { cleaners }\end{array}$ & N/A & $\mathbf{N} / \mathbf{A}$ & N/A \\
\hline \multirow[t]{13}{*}{ K. Thermal } & $\begin{array}{l}\text { 1. Bunsen burner/ } \\
\text { hot plates }\end{array}$ & $\mathbf{N} / \mathbf{A}$ & $\mathbf{N} / \mathbf{A}$ & N/A \\
\hline & $\begin{array}{l}\text { 2. Electrical } \\
\text { equipment }\end{array}$ & $\begin{array}{l}\text { Unit heaters and space heaters } \\
\text { Electrical motors and pumps }\end{array}$ & $\begin{array}{l}\text { Space heaters } \\
\text { Eloctrical furnaces heaters } \\
\text { Switchgear room } \\
\text { Electrical motors and pumps }\end{array}$ & None' \\
\hline & $\begin{array}{l}\text { 3. Furnaces/ } \\
\text { boilers/heater }\end{array}$ & Boiler package in $\mathrm{KE} / \mathrm{KW}$ & Unit heaters & None $^{1}$ \\
\hline & 4. Steam lines & N/A & N/A & N/A \\
\hline & $\begin{array}{l}\text { 5. Welding } \\
\text { torch/arc }\end{array}$ & Construction and maintenance & $\begin{array}{l}\text { Construction and } \\
\text { maintenance }\end{array}$ & None ${ }^{1}$ \\
\hline & $\begin{array}{l}\text { 6. Diesel unita/fire } \\
\text { box/exhaust } \\
\text { line }\end{array}$ & Truck exhaust & Truck exhaust & None ${ }^{1}$ \\
\hline & $\begin{array}{l}\text { 7. Radioactive } \\
\text { decay heat }\end{array}$ & $\begin{array}{l}\text { Decay heat from fuel } \\
\text { Cask-MCO }\end{array}$ & $\mathbf{N} / \mathbf{A}$ & Yes \\
\hline & $\begin{array}{l}\text { 8. Exposed } \\
\text { component }\end{array}$ & $\mathbf{N} / \mathbf{A}$ & N/A & N/A \\
\hline & 9. Power tools & High speed grinders & High speod grinders & None ${ }^{1}$ \\
\hline & 10. Convective & $\begin{array}{l}\text { Motors, lighting, instrumentation, } \\
\text { control panels }\end{array}$ & $\begin{array}{l}\text { Motors, lighting, } \\
\text { instrumentation, control } \\
\text { panels }\end{array}$ & None' \\
\hline & 11. Solar & N/A & N/A & N/A \\
\hline & 12. Cryogenic & N/A & N/A & $\mathbf{N} / \mathbf{A}$ \\
\hline & 13. Other & $\mathbf{N} / \mathbf{A}$ & N/A & $\mathbf{N} / \mathbf{A}$ \\
\hline
\end{tabular}


HNF-3960 REV 2

Table B-1. Hazard Baseline - Energy Checklist (13 Sheets).

\begin{tabular}{|c|c|c|c|c|}
\hline $\begin{array}{l}\text { Type of } \\
\text { Hazard }\end{array}$ & Form of Hazard & 105 KE/KW Basin & $\begin{array}{l}\text { Other Supporting } \\
\text { Structures, Systems, and } \\
\text { Components }\end{array}$ & $\begin{array}{l}\text { Hazards } \\
\text { Requiring } \\
\text { Additional } \\
\text { Analyois }\end{array}$ \\
\hline \multirow{12}{*}{$\begin{array}{l}\text { L. Explosive } \\
\text { Pyrophoric }\end{array}$} & 1. Caps & N/A & N/A & N/A \\
\hline & 2. Primer cord & N/A & N/A & N/A \\
\hline & 3. Dynamite & N/A & N/A & $\mathbf{N} / \mathbf{A}$ \\
\hline & 4. Serub chemicals & N/A & N/A & N/A \\
\hline & 5. Dusts & N/A & N/A & N/A \\
\hline & $\begin{array}{l}\text { 6. Hydrogen } \\
\text { (including } \\
\text { bettery banks } \\
\text { and water } \\
\text { decomposition) }\end{array}$ & $\begin{array}{l}\text { Knockout pots } \\
\text { Canisters/decapping } \\
\text { Basin sludge } \\
\text { Particulate settler } \\
\text { Cask-MCO } \\
\text { Annular filtor vessels }\end{array}$ & $\begin{array}{l}\text { Battery rooms cach provided } \\
\text { with dedicated fans to ensure } \\
\text { H gas is released. } \\
\mathrm{DXCs}(183.1 \mathrm{KW})\end{array}$ & Yes \\
\hline & 7. Gases, other & N/A & N/A & N/A \\
\hline & 8. Nitrates & N/A & N/A & N/A \\
\hline & 9. Peroxides & $\begin{array}{l}\text { Hydrogen peroxide used to control } \\
\text { algee in basins }\end{array}$ & N/A & None' \\
\hline & $\begin{array}{l}\text { 10. Pu and } U \\
\text { metals }\end{array}$ & $\begin{array}{l}\text { Fuel assemblies, fuel scrap, } \\
\text { uranium hydrides, knockout pots, } \\
\text { settlers, annular filter vessel }\end{array}$ & N/A & Yes \\
\hline & 11. Sodium & N/A & N/A & N/A \\
\hline & 12. Other & N/A & N/A & N/A \\
\hline \multirow{7}{*}{$\begin{array}{l}\text { M. Hazardous } \\
\text { Material }\end{array}$} & 1. Alkali metals & N/A & N/A & N/A \\
\hline & 2. Asphyxiants & $\begin{array}{l}\text { Basin water (drowning) } \\
\text { Vehicle exhaust } \\
\text { Inert gases for } \\
\text { welding/maintenance }\end{array}$ & $\begin{array}{l}\text { Clearwells (underground } \\
\text { water storage reservoirs with } \\
\text { depth } \sim 20 \mathrm{ft} \text { water) } \\
\text { (drowning) }\end{array}$ & None' \\
\hline & 3. Biologicals & Spiders/insocts/snakes/mice & Spiders/insects/snakes/mice & None' \\
\hline & 4. Carcinogens & PCBs (in sludge) & $\begin{array}{l}\mathrm{PCB} \text { (possible residual from } \\
\text { transformers) }\end{array}$ & None $^{3}$ \\
\hline & 5. Corrosives & Battery acid & $\begin{array}{l}\text { Vehicle battery acid } \\
\text { Battery room }\end{array}$ & None ${ }^{1}$ \\
\hline & 6. Asbestos & Building/piping & Building/piping & None \\
\hline & 7. Oxidizers & Decon agents & Decon agents & None $^{1}$ \\
\hline
\end{tabular}


Table B-1. Hazard Baseline - Energy Checklist (13 Sheets).

\begin{tabular}{|c|c|c|c|c|c|}
\hline & $\begin{array}{l}\text { Type of } \\
\text { Heward }\end{array}$ & Form of Hazard & $10 S \mathrm{KE} / \mathrm{KW}$ Bamin & $\begin{array}{c}\text { Other Supportins } \\
\text { Structures, Systems, and } \\
\text { Components }\end{array}$ & $\begin{array}{l}\text { Henards } \\
\text { Requiring } \\
\text { Additional } \\
\text { Andyois }\end{array}$ \\
\hline \multirow{5}{*}{\multicolumn{2}{|c|}{$\begin{array}{l}\text { M. Hazardous } \\
\text { Material } \\
\text { cont. }\end{array}$}} & $\begin{array}{l}\text { 8. Dusts and } \\
\text { particulates }\end{array}$ & $\begin{array}{l}\text { Sand and dust } \\
\text { Volcanic ash } \\
\text { Construction and demolition }\end{array}$ & $\begin{array}{l}\text { Sand and dust } \\
\text { Volcanic ash } \\
\text { Construction and demolition }\end{array}$ & None' \\
\hline & & $\begin{array}{l}\text { 9. Beryllium and } \\
\text { compounds }\end{array}$ & In-fuel braze rings (underwater) & Potentially 1706-KE & None ${ }^{3}$ \\
\hline & & $\begin{array}{l}\text { 10. Chlorine and } \\
\text { compounds }\end{array}$ & N/A & N/A & $\mathbf{N} / \mathbf{A}$ \\
\hline & & 11. Heavy metal & Pu, U, lead, lead paint & Load, lead paint & None \\
\hline & & 12. Other & $\mathbf{N} / \mathbf{A}$ & $\begin{array}{l}\text { Sodium hydrochloride, } \\
\text { aluminum sulfate }\end{array}$ & None \\
\hline & \multirow{9}{*}{$\begin{array}{l}\text { Natural } \\
\text { Phenomena }\end{array}$} & 1. Earthquake & Design basis earthquake & Design basis earthquake & Yes \\
\hline & & 2. Flood & N/A & Yos & Yes \\
\hline & & 3. Lightning & Yes & Yes. & Yes \\
\hline & & 4. Rain & Yes & Yes & Yos \\
\hline & & $\begin{array}{l}\text { 5. Snow, froezing } \\
\text { weather }\end{array}$ & Yes & Yes & Yes \\
\hline & & 6. Straight wind & Yes & Yes & Yes \\
\hline & & 7. Dust devil & (Covered by straight wind) & (Covered by straight wind) & N/A \\
\hline & & 8. Tomado & (Covered by straight wind) & (Covered by straight wind) & N/A \\
\hline & & 9. Ashfall & Yes & $\mathbf{N} / \mathbf{A}$ & Yes \\
\hline \multirow{4}{*}{\multicolumn{2}{|c|}{$\begin{array}{l}\text { P. External } \\
\text { Events }\end{array}$}} & 1. Explosion & N/A & $\mathbf{N} / \mathbf{A}$ & N/A \\
\hline & & 2. Fire & Yes & Yes & Yes \\
\hline & & $\begin{array}{l}\text { 3. Events at other } \\
\text { sites }\end{array}$ & Yes & Yes & Yes \\
\hline & & 4. Loss of power & Yos & Yes & Yes \\
\hline \multirow{4}{*}{ R. } & Vehicles in & 1. Airplane & Commercial, general, and military & N/A & Yes \\
\hline & & 2. Helioopter & Commercial, general, and military & N/A & Yes \\
\hline & & 3. Train & $\mathbf{N} / \mathbf{A}$ & N/A & $\mathbf{N} / \mathbf{A}$ \\
\hline & & 4. Truck/bua/car & $\begin{array}{l}\text { Only authorized vehicle travel } \\
\text { permitted within K Basin. Only } \\
\text { two public roads that cross the } \\
\text { Hanford Site. Remaining roads are } \\
\text { restricted access roads. }\end{array}$ & N/A & None $^{1}$ \\
\hline
\end{tabular}


HNF-3960 REV 2

Table B-1. Hazard Baseline - Energy Checklist (13 Sheets).

\begin{tabular}{|l|l|l|l|c|}
\hline \multicolumn{1}{|c|}{$\begin{array}{c}\text { Type of } \\
\text { Hazard }\end{array}$} & Form of Hazard & \multicolumn{1}{|c|}{$105 \mathrm{KWE} / \mathrm{KW}$ Basin } & $\begin{array}{c}\text { Hazards } \\
\text { Other Supporting } \\
\text { Structurea, Syotems, and } \\
\text { Components }\end{array}$ & $\begin{array}{c}\text { Requiring } \\
\text { Additional } \\
\text { Analyalo }\end{array}$ \\
\hline $\begin{array}{c}\text { R. Vehicles in } \\
\text { Motion } \\
\text { (external to } \\
\text { facility) } \\
\text { cont. }\end{array}$ & 5. Other & River barge and boat traffic & N/A & None $^{1}$ \\
\hline
\end{tabular}

1 Considered to be a standard industrial hazard.

2 Considered to be a standard fire hazard covered by HNF-SD-SNF-FHA-001, 1999, Five Hazards Analysis for the K Basins Facilities at $100 \mathrm{~K}$ Area (Rev. 1, Fluor Daniel Henford, Inc., Richland, Washington), and not considered for further evaluation.

3 Considered to be a toxicological hazard, but when radiological consequences are controlled to meet radiological limits and guidelines, toxicological consequences will also be below toxicological guidelines.

\begin{tabular}{|c|c|c|}
\hline AC & $=$ & alternating current \\
\hline DC & $=$ & direct current \\
\hline GFI & $=$ & ground fault interruptor \\
\hline IPSS & $=$ & immersion pail support structure \\
\hline IWTS & $=$ & Integrated Water Treatment System \\
\hline IXC & $=$ & ion-exchange columnm \\
\hline $\mathbf{D X M}$ & $=$ & ion-exchange module \\
\hline KE & $=$ & K East \\
\hline KW & $=$ & K West \\
\hline $\mathrm{MCO}$ & $=$ & multi-canister overpack \\
\hline MLS & $=$ & MCO loading system \\
\hline N/A & $=$ & not applicable \\
\hline NLOP & $=$ & north loadout pit \\
\hline PCB & $=$ & polychlorinsted biphenyl \\
\hline $\mathrm{PCM}$ & $=$ & primary clean machine \\
\hline PV & $=$ & pressure-volume \\
\hline SCBA & $=$ & self-contained breathing apparatus \\
\hline
\end{tabular}




\section{HNF-3960 REV 2}

This page intentionally left blank. 
HNF-3960 REV 2

Attachment C

HAZARD ANALYSIS WORKSHEET 


\section{HNF-3960 REV 2}

This page intentionally left blank 


\section{HAZARD ANALYSIS WORKSHEET}

The following is a description of the Hazard Analysis Worksheet columns:

\section{Column}

Number

\section{Description of Content}

Assigns a numeric identifier to a specific accident.

2 Identifies the energy source "hazard" from the energy source checklist in Appendix B.

Briefly describes the hazardous condition.

Identifies the cause of the hazardous condition (typically human error, equipment failure, or natural phenomena, or all three).

Describes the potential accident associated with the hazard energy and hazardous condition.

Contains a qualitative assessment of the result of the potential accident.

7 Identifies equipment or administrative controls credited to prevent consequences of the accident.

8 Contains a qualitative estimate of the frequency of the event, divided into two sections, without and with credited prevention measures.

9 Identifies equipment or administrative controls credited to mitigate consequences of the accident.

10 Contains a qualitative estimate of the consequence of the event divided into two sections, without and with credited mitigation measures.

11 Identifies other equipment or controls that provide additional measures for prevention or mitigation of the accident, which are not credited in the accident analysis but do provide defense-in-depth protection.

12 Provides risk ranking (from Figure 3-3 in DOE-STD-3009-94) and accident identification for cross-reference. 
HNF-3960 REV 2

This page intentionally left blank

hnf-3960.r2 
HNF-3960 REV 2

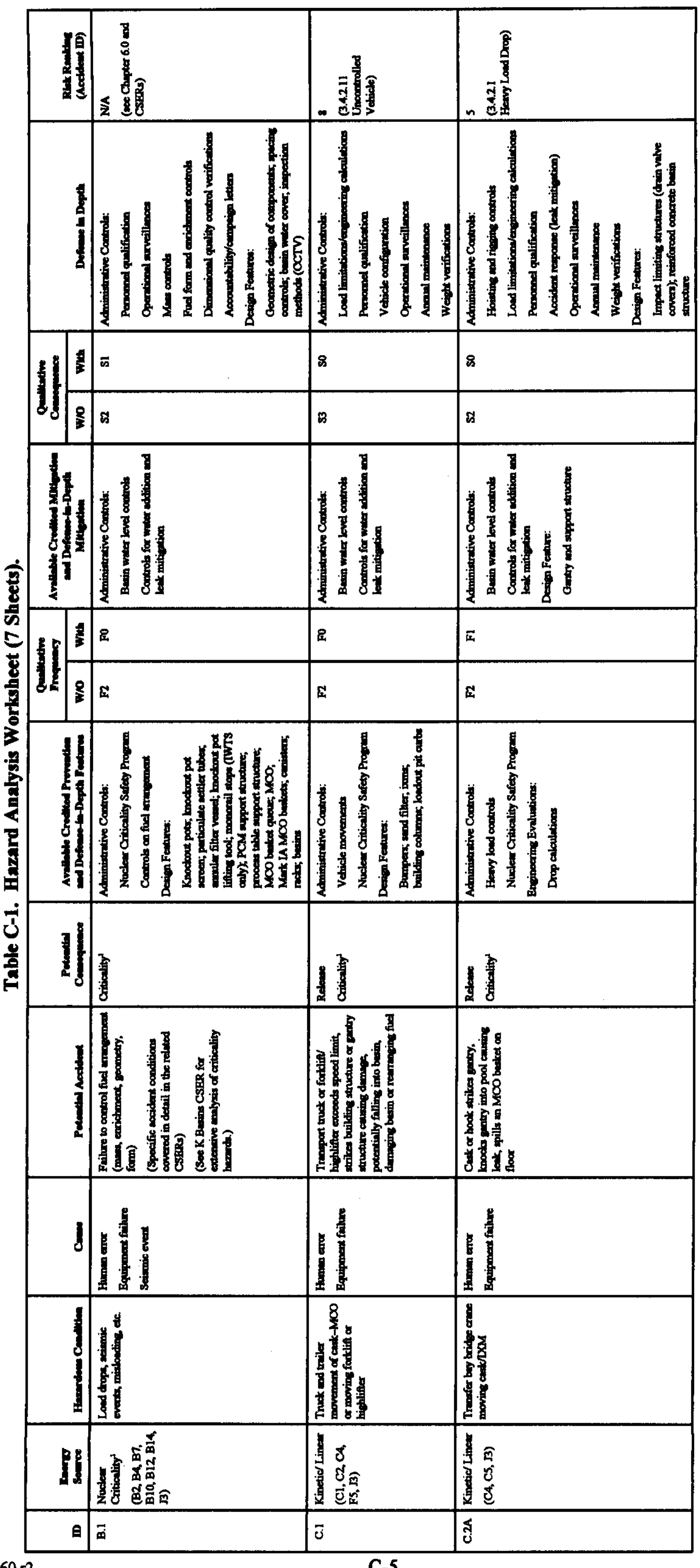


HNF-3960 REV 2

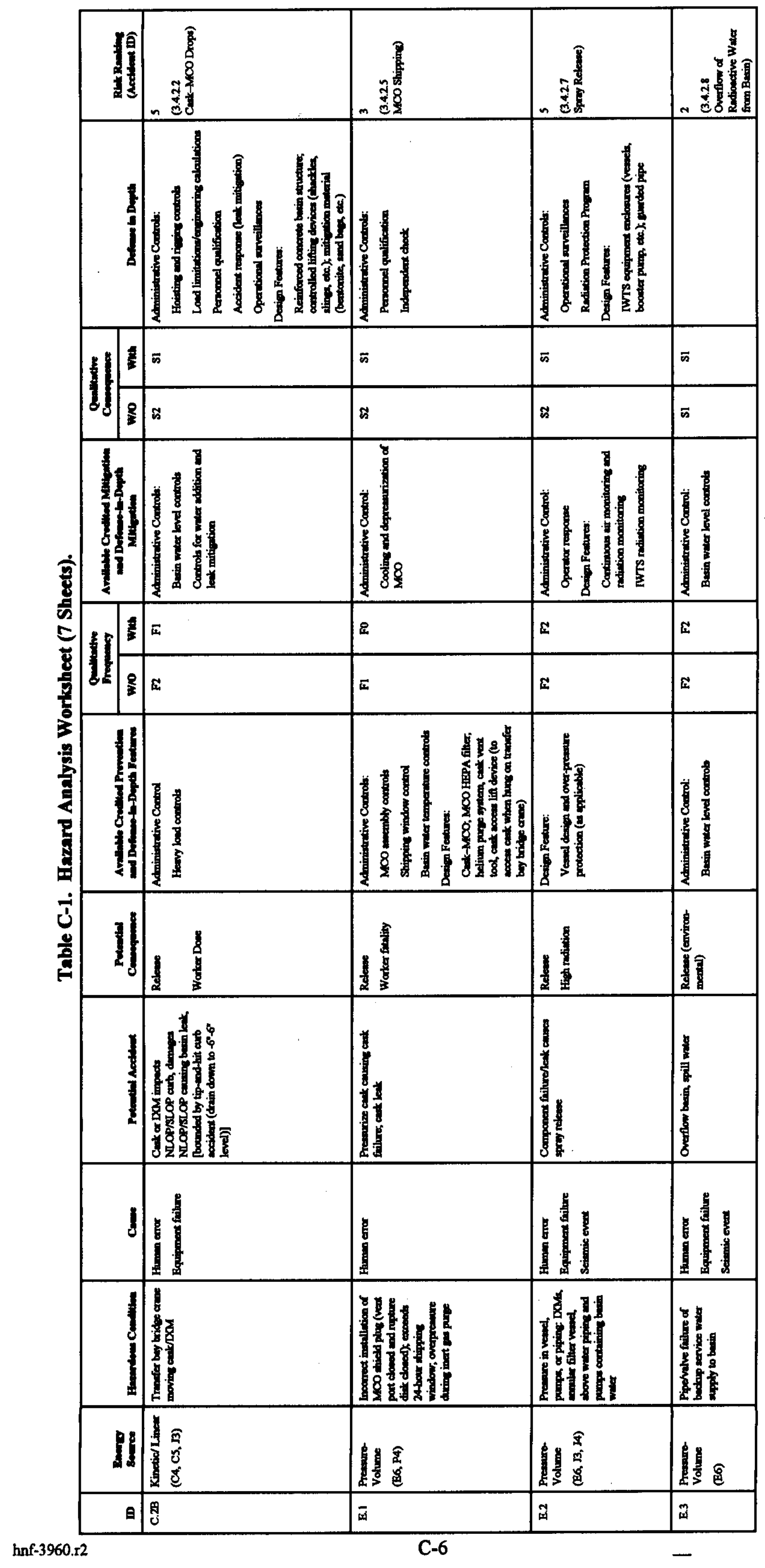


HNF-3960 REV 2

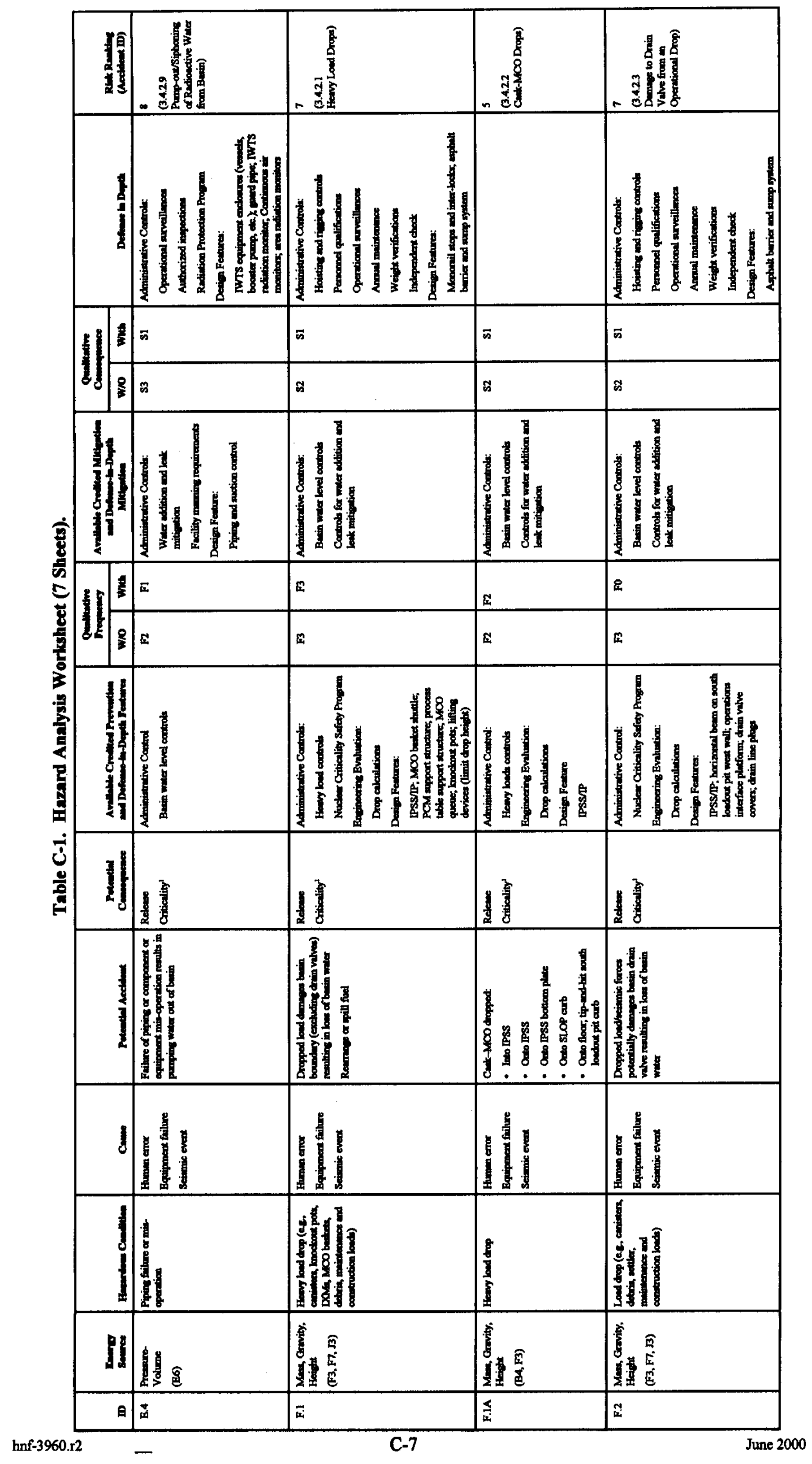


HNF-3960 REV 2

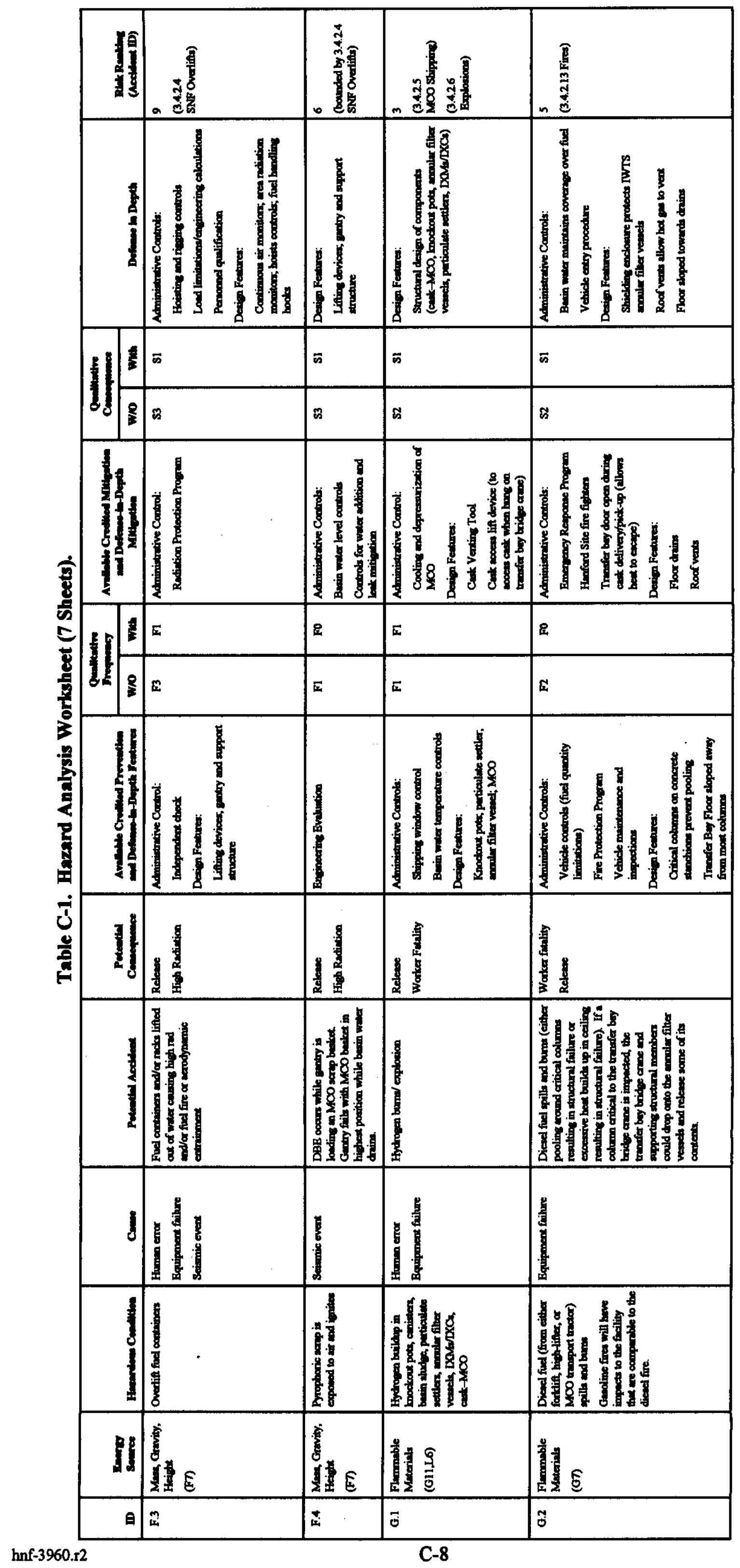


HNF-3960 REV 2

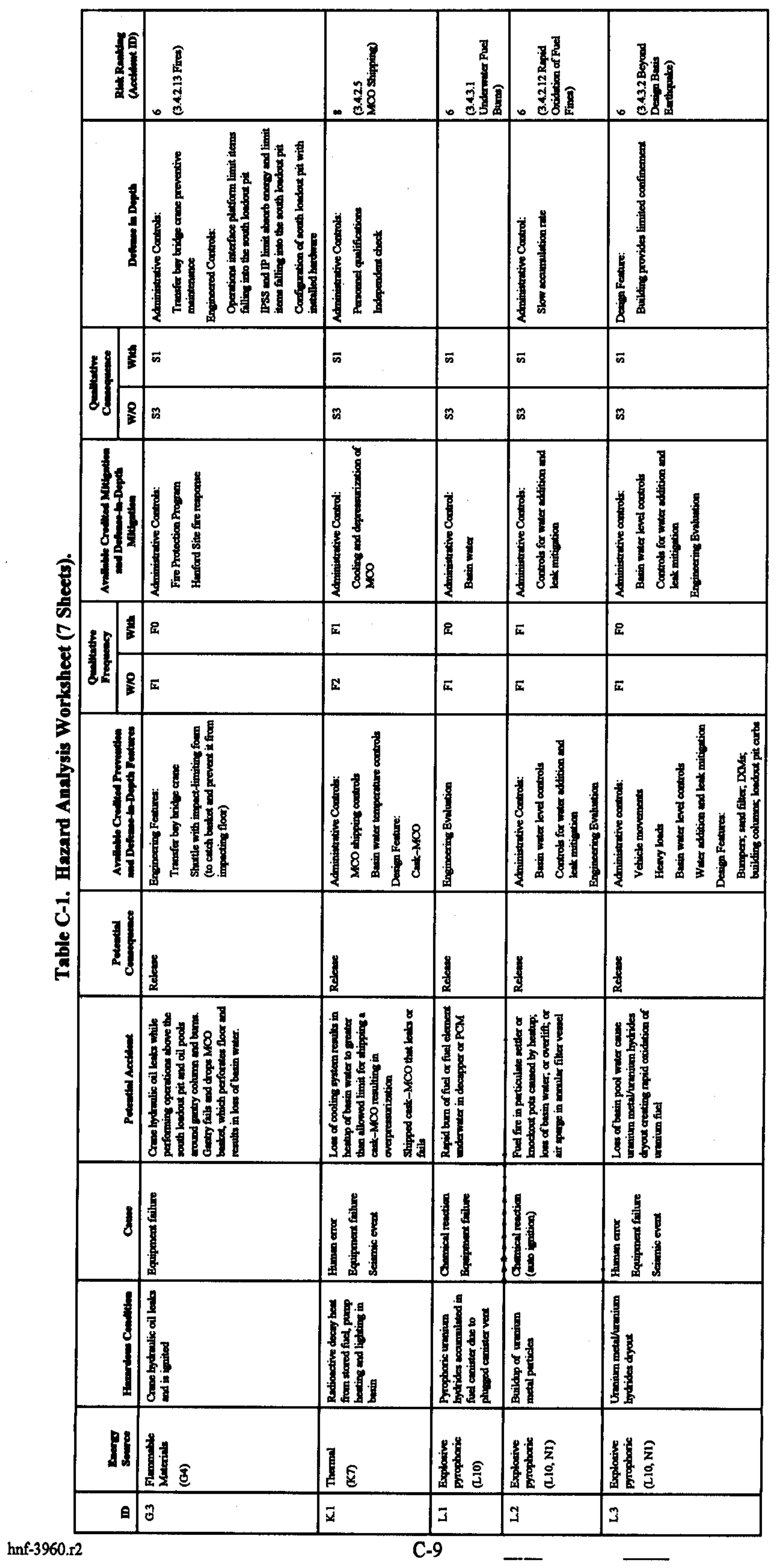


HNF-3960 REV 2

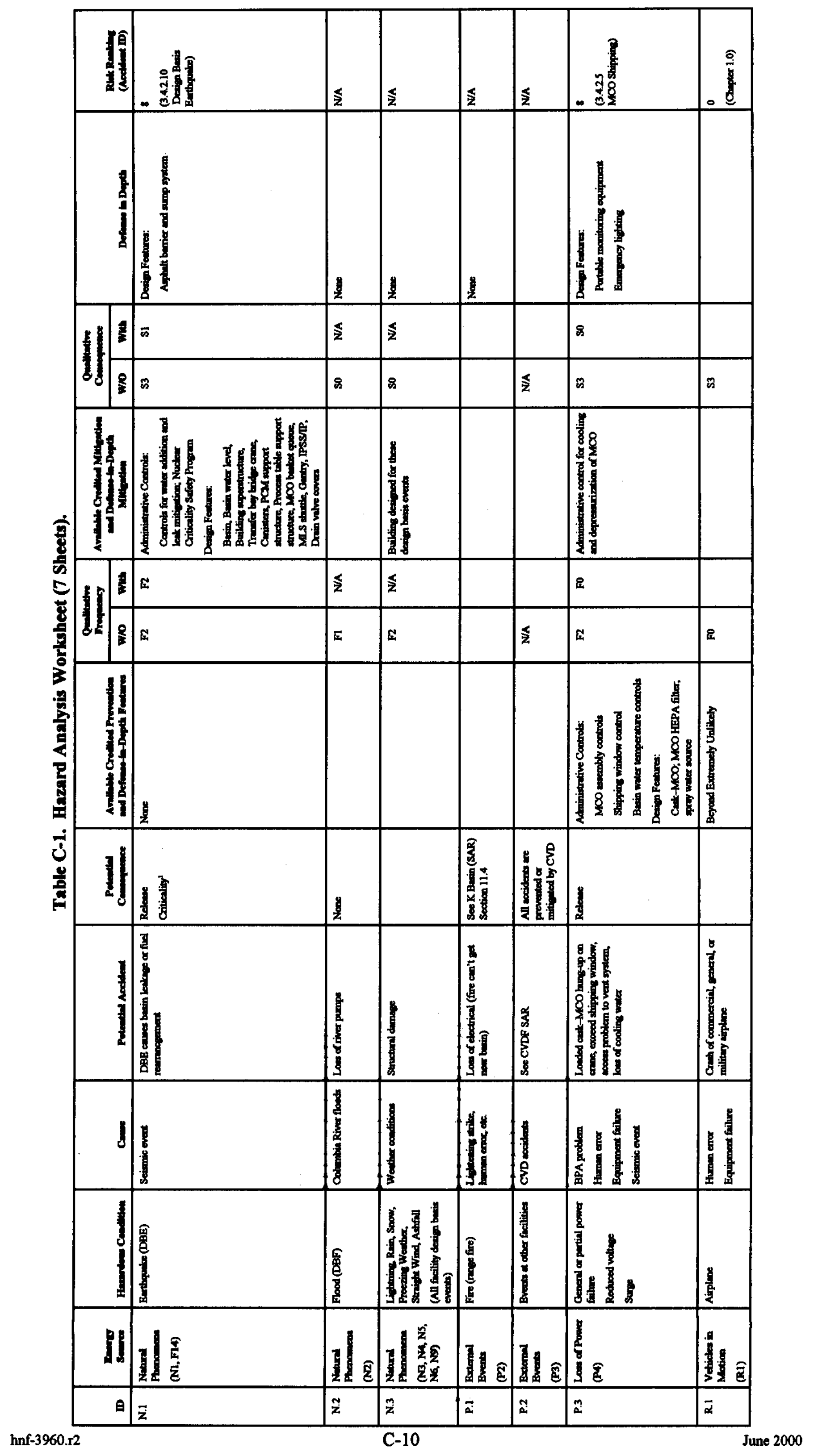




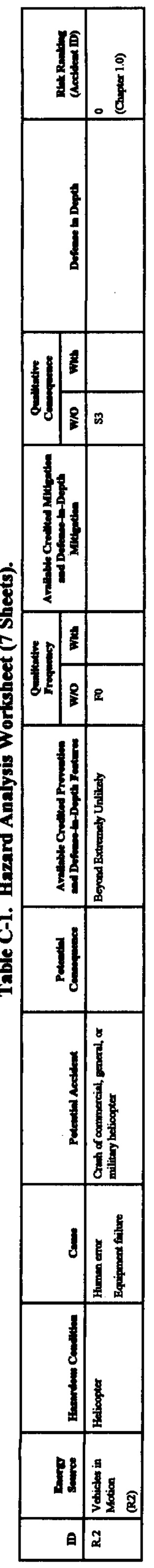

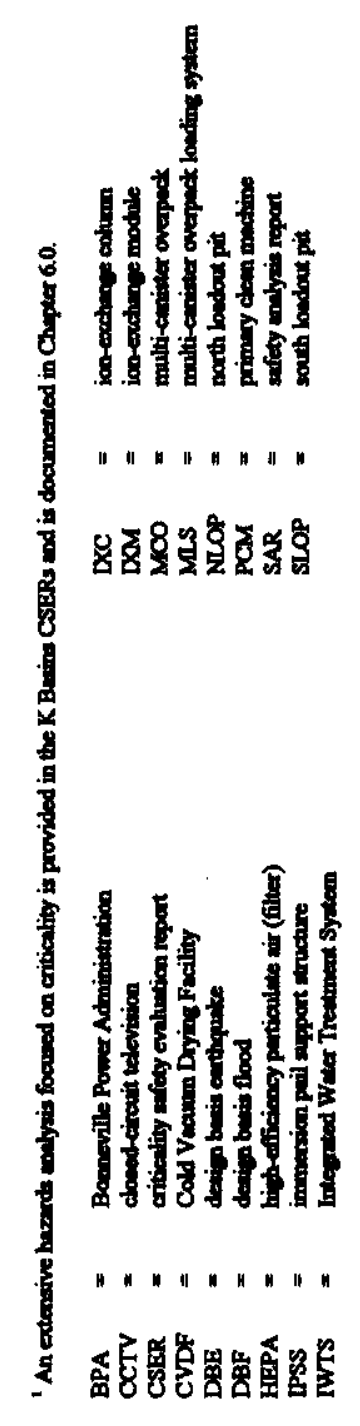

C-11 
HNF-3960 REV 2

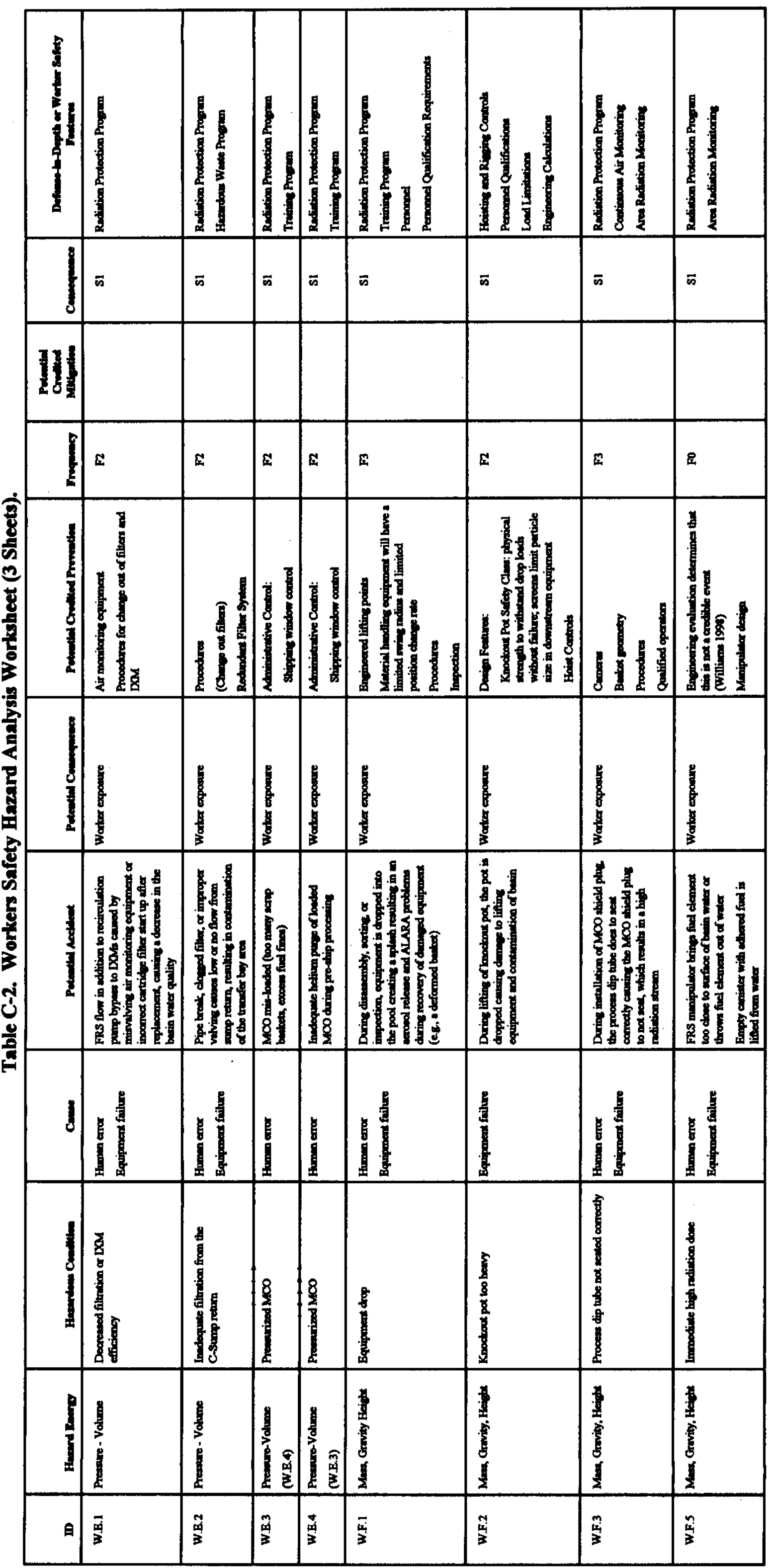


HNF-3960 REV 2

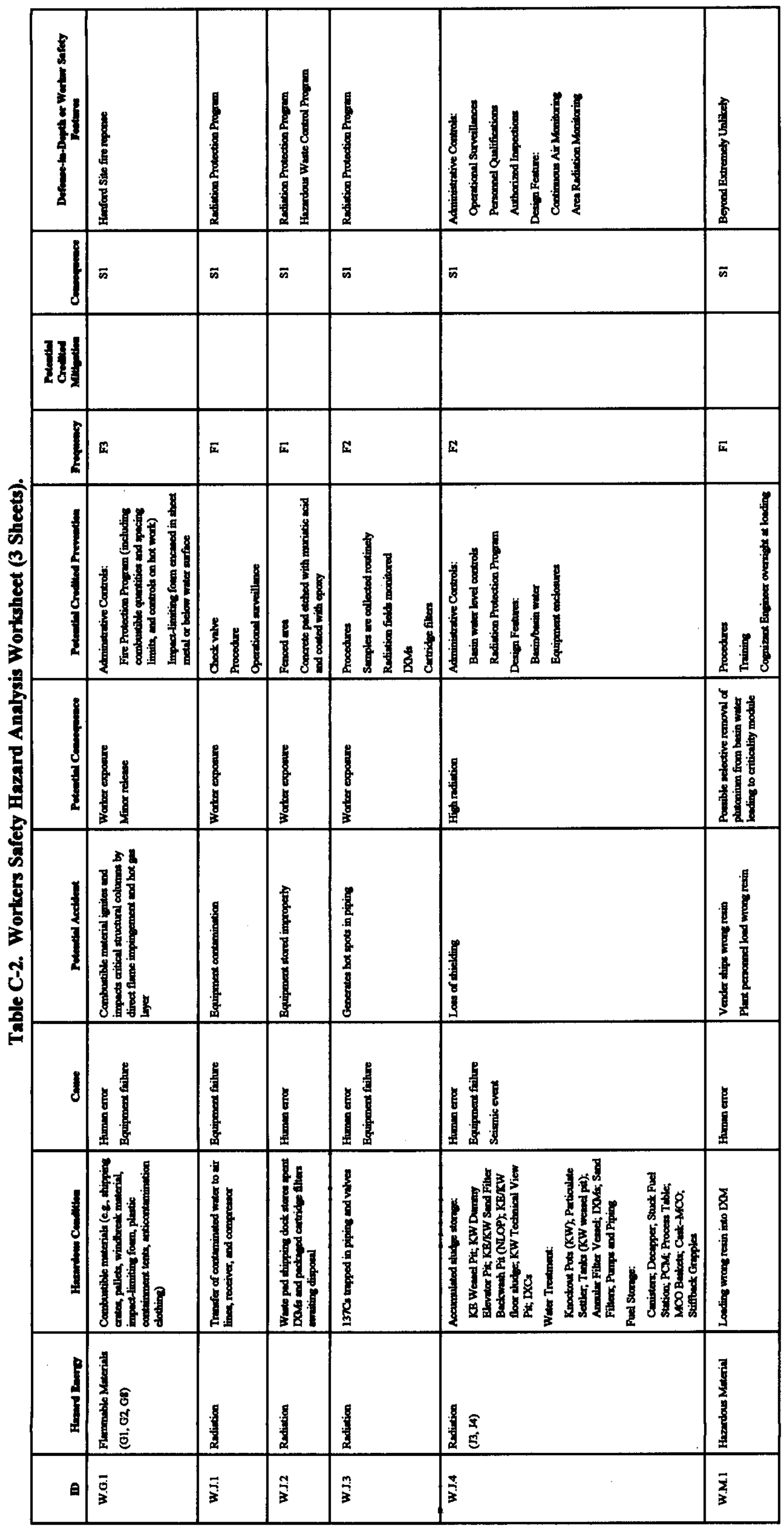


HNF-3960 REV 2

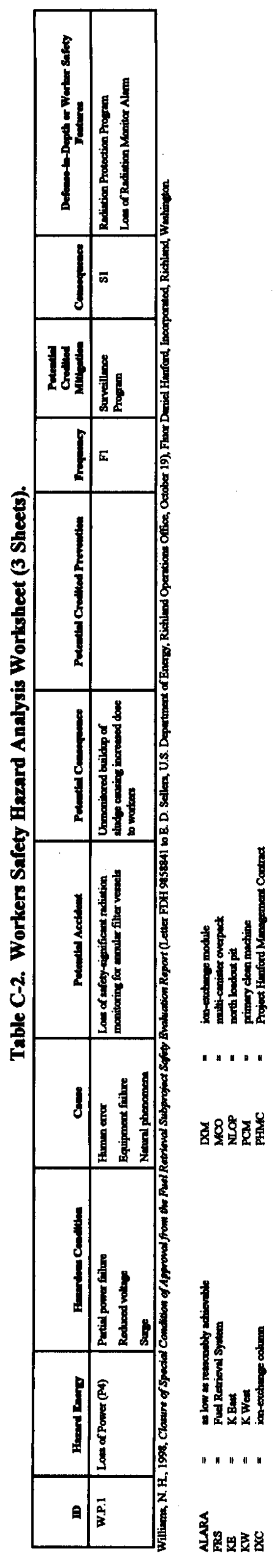


HNF-3960 REV 2

Attachment D

K BASIN HAZARD ANALYSIS TEAM MEMBERS 


\section{HNF-3960 REV 2}

This page intentionally left blank 


\section{Attachment D}

\section{K BASIN HAZARD ANALYSIS TEAM MEMBERS}

This hazard analysis was originally prepared by combining the hazard analyses from several Spent Nuclear Fuel Project subprojects with the existing K Basins hazard analysis. Subsequently it has been updated to include other projects and activities. This attachment documents the personnel who have participated in developing and updating this hazard analysis.

The initial K Basins Hazard analysis was developed by the following personnel:

Gail Chaffee (100 K Basins Nuclear Safety)

Bob Meichle (K Basins Nuclear Safety Subject Matter Expert)

Steve Peck (Fuel Retrieval System Nuclear Safety Subject Matter Expert)

Lynn Semmens (Integrated Water Treatment System Nuclear Safety Subject Matter Expert)

Roger Webb (Cask Loadout System Nuclear Safety Subject Matter Expert).

The following personnel were involved in the hazard analysis for the canister cleaning equipment:

Christine Bullock (SNF RAD Con)

Dave Bullock (KW Operations)

Jerry Chiaramonte (Cogema, Project Manager)

Denise Clements (Cogems, Quality Assurance)

Carol Farwick (Vista Engineering)

John Irons (Cogema, Mechanical Lead)

Chris Lucas (Operations Support Manager)

Frank Muller (Fluor Hanford, Project Manager)

Dick Nelson (MCE, Greenhouse Design)

Phil Ohl (Design Review Chairman)

Steve Peck (MCE, SNF Nuclear Safety)

Don Precechtel (Canister Cleaning Design Authority)

Mark Rickenback (Cogema Engineering)

Chris Thompson (BSI, CCS). 
HNF-3960 REV 2

This page intentionally left blank 